\title{
A stress tensor discontinuity-based immersed boundary-lattice Boltzmann method
}

\author{
Kosuke Suzuki $^{\mathrm{a}, *}$, Masato Yoshino ${ }^{\mathrm{a}, \mathrm{b}}$ \\ ${ }^{a}$ Institute of Engineering, Academic Assembly, Shinshu University, Nagano 380-8553, \\ JAPAN \\ ${ }^{b}$ Institute of Carbon Science and Technology, Interdisciplinary Cluster for Cutting Edge \\ Research, Shinshu University, Nagano 380-8553, JAPAN
}

\begin{abstract}
We propose an immersed boundary-lattice Boltzmann method using the discontinuity of the stress tensor. In the immersed boundary method, the body force which is applied to enforce the no-slip boundary condition is equivalent to the discontinuity of the stress tensor across the boundary. In the proposed method, the boundary is expressed by Lagrangian points independently of the background lattice points, and the discontinuity of the stress tensor is calculated on these points from desired particle distribution functions which satisfy the no-slip boundary condition based on the bounce-back scheme. By using this method, we can obtain the force locally acting on the boundary from the stress tensor of one side of the fluids divided by the boundary, and there is no need to consider the internal mass effect in calculating the total force and torque acting on the boundary. To our best knowledge, the present method is the first one which enables us to calculate the stress tensor on the boundary in the class of the diffusive interface method. In order to validate the present method, we apply it to simulations of typical moving-boundary problems, i.e., a Taylor-Couette flow, an oscillating circular cylinder in a stationary fluid, the sedimentation of an elliptical cylinder, and the sedimentation of a sphere. As a result, the present method has the first-order spatial accuracy and has a good agreement with other
\end{abstract}

\footnotetext{
* Corresponding author

Email addresses: kosuzuki@shinshu-u.ac.jp (Kosuke Suzuki), masato@shinshu-u.ac.jp (Masato Yoshino)
}

Preprint submitted to Computers \& Fluids

February 3, 2021 
numerical and experimental results. In addition, we discuss two problems of the present method, i.e., penetration and spurious oscillation of local force, and a possible remedy for them.

Keywords: Immersed boundary method, Lattice Boltzmann method, Moving-boundary flow, Stress tensor discontinuity

\section{Introduction}

One of the important issues in computational fluid dynamics is to simulate moving-boundary flows efficiently and accurately. The simplest way is to approximate the boundary by staircase-like steps in a fixed Cartesian grid (e.g.,

5 early investigations into particulate suspensions [1, 2]). In applying the approximation to moving-boundary flows, however, it is required to construct new staircase-like steps in each time step, and the complicated procedure does not balance with its low accuracy. Other ways are body-fitted or unstructuredgrid methods in which the grid conforms to the boundary. These methods can express arbitrary boundaries accurately and has traditionally been used for moving-boundary flows. However, due to re-meshing procedures, the algorithms of the methods are generally complicated, and also the computation costs are expensive. Recently, the immersed boundary method (IBM), which was proposed by Peskin $[3,4]$ in 1970s in order to simulate blood flows in the heart, has been reconsidered as an efficient method for simulating moving-boundary flows on a fixed Cartesian grid. In the IBM, it is assumed that the boundary is regarded as an infinitely thin shell, an incompressible viscous fluid is filled in both inside and outside of the boundary, and the no-slip condition on the boundary is satisfied by body force applied only near the boundary. The way to determine the body force is the key concept of the IBM, and a lot of variations of the IBM with different ways to determine the body force have been proposed as reviewed by Mittal and Iaccarino [5].

In general, the body force is determined for satisfying the no-slip condition. Depending on the variations of the IBM, there are many ways to incorporate the 
no-slip condition into the body force. From this viewpoint, the variations of the IBM can be roughly classified into the following two categories: displacementbased IBM and velocity-based IBM. In the displacement-based IBM, which includes the original IBM [3,4] and its modification for rigid boundaries [6], the displacements of boundary Lagrangian points are determined by the no-slip condition, and then the body force is determined as the surface stress corresponding to the displacement derived from a constitutive law. In the velocity-based IBM, the desired velocity on and/or near the boundary is determined by the no-slip condition, and then the body force is determined by the difference between the desired velocity and the flow velocity without regard to the boundary. This category includes a large class of the variations of the IBM, e.g., the volume penalization method firstly introduced by Arquis and Caltagirone [7], the virtual boundary method proposed by Goldstein et al. [8], the direct forcing method firstly introduced by Mohd-Yusof [9], the fictitious domain method proposed by Glowinski et al. [10], the cut-cell method proposed by Udaykumar et al. [11], 40 the ghost cell method firstly implemented by Majumdar et al. [12], the IBM with direct forcing using the Peskin's regularized delta function [13] proposed by Uhlmann [14], and their variations and improvements.

In the present study, we propose a new method which does not belong to the above two categories. The proposed method is based on the discontinuity of the 45 stress tensor across the boundary (the relation between the body force and the discontinuity of the stress tensor is explained in Section 2). If the stress tensors which satisfy the no-slip condition can be obtained, we can calculate the body force from their discontinuity. However, it is almost impossible to calculate them before the pressure and velocity fields are determined, and therefore there has been no method based on this concept. It should be noted that this concept has been utilized in the immersed interface method (IIM) [15] exceptionally. Even in the IIM, however, the body force is determined independently of it (e.g., the IIM proposed by Lee and Leveque [16] is categorized to the displacement-based IBM and that recently proposed by $\mathrm{Li}$ et al. [17] is categorized to the velocitybased IBM), and the way to distribute the body force is determined on the basis 
of this concept. Then, how can we obtain the discontinuity of the stress tensor which satisfies the no-slip condition in advance?

We can find a clue to answer the above question from the momentum exchange-based immersed boundary-lattice Boltzmann method (IB-LBM) [18]. 60 In this method, the pressure and velocity fields are calculated by the lattice Boltzmann method (LBM), where finite-difference equations for particle distribution functions (PDFs) of a modeled gas (which is composed of identical particles whose velocities are restricted to a finite set of vectors [19]) are solved, and the macroscopic variables such as the pressure and velocity are calculated by the moments of the PDFs. The procedure to determine the body force in the momentum exchange-based IB-LBM is as follows: (i) the desired PDFs which satisfy the no-slip condition on the boundary are calculated by using the bounceback scheme [20], and (ii) the body force is determined from the amount of the momentum exchange between the desired PDFs and those without regard to the boundary. Therefore, this method does not belong to the above two categories of the variations of the IBM, too. It should be noted that this method can be implemented only in the framework of the LBM, since the PDF has an essential role in determining the body force. Recent improvements and applications of the momentum exchange-based IB-LBM can be found in Refs. [21-24].

75 In the present method, the desired PDFs in the above procedure (i) are utilized for calculating the stress tensors inside and outside the boundary by using the relation between the stress tensor and the PDFs obtained from the asymptotic analysis [25], and then the body force is determined from the discontinuity of the stress tensor. The advantage in obtaining the stress tensor instead of calculating the momentum exchange is to be able to calculate the local stress acting on the boundary from the external fluid outside the boundary independently of the state of the internal fluid inside the boundary. In addition, there is no need to consider the internal mass effect [26] in calculating the total force and torque acting on the boundary. The present method can be implemented only in the framework of the LBM, too. It should be noted that the present method is categorized into the diffusive interface method, in which the Peskin's 
regularized delta function [13] is used for distributing the body force on the lattice points near the boundary, although the discontinuity of the stress tensor is sharply determined. To our best knowledge, the present method is the first one which enables us to calculate the stress tensor on the boundary in the class of the diffusive interface method.

The paper is organized as follows. In Section 2, we explain the relation between the body force and the discontinuity of the stress tensor. In Section 3, we describe the present numerical method. In Section 4, we validate it through benchmark problems including two- and three-dimensional flows with moving boundaries. In Section 5, we discuss some problems of the present method and a possible remedy for them. We finally conclude in Section 6 .

\section{Relation between the body force and the discontinuity of the stress tensor}

First of all, we assume that an incompressible viscous fluid is filled both inside and outside the boundary. Supposing that the fluids separated by the boundary be Fluids \#1 and \#2 only for the purpose of convenience, these fluids should receive stresses $\boldsymbol{P}^{[1]}$ and $\boldsymbol{P}^{[2]}$ through a small surface element $d S$ of the boundary, respectively (see Fig. 1). In the IBM, the stresses are distributed as the body force $\boldsymbol{g}$ to a small volume $d V$ near the surface element as follows:

$$
\boldsymbol{g} d V=\left(\boldsymbol{P}^{[1]}+\boldsymbol{P}^{[2]}\right) d S
$$

where we assume that the surface element $d S$ is so small that $\boldsymbol{P}^{[1]}$ and $\boldsymbol{P}^{[2]}$ are constant in $d S$, and that the volume element $d V$ is so small that $\boldsymbol{g}$ is constant in $d V$. When written out by using the corresponding stress tensors, Eq. (1) becomes

$$
\boldsymbol{g} d V=-\left(\boldsymbol{\sigma}^{[1]}-\boldsymbol{\sigma}^{[2]}\right) \cdot \boldsymbol{n} d S
$$

where $\boldsymbol{\sigma}^{[\ell]}(\ell=1,2)$ is the stress tensor acting on the surface element from Fluid $\# \ell$ and $\boldsymbol{n}$ is the unit normal vector on the surface element pointing to Fluid \#1. Therefore, the body force in the IBM is equivalent to the discontinuity of the stress tensor across the boundary. 


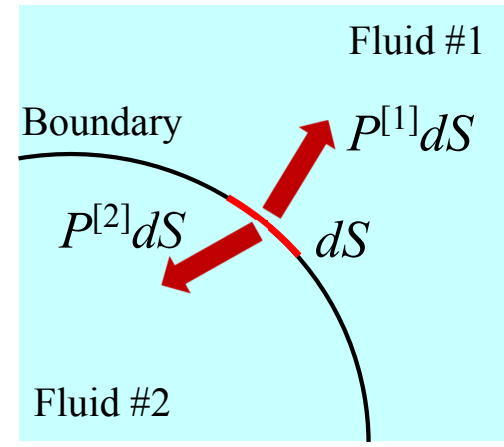

Figure 1: Illustration of stresses acting on Fluids \#1 and \#2 through a small surface element of the boundary.

\section{Stress tensor discontinuity-based immersed boundary-lattice Boltz- mann method}

\subsection{Lattice Boltzmann method}

In the LBM, a modeled gas, which is composed of identical particles whose velocities are restricted to a finite set of vectors, is considered [19]. Twodimensional lattice with nine velocity vectors (D2Q9 model) and three-dimensional lattice with fifteen velocity vectors (D3Q15 model) are used in the present study. In the following, we use non-dimensional variables normalized by a characteristic length $\hat{H}_{0}$, a characteristic particle speed $\hat{c}_{\mathrm{p}}$, a characteristic time scale $\hat{t}_{0}=\hat{H}_{0} / \hat{U}_{0}$ (where $\hat{U}_{0}$ is a characteristic flow speed), and a fluid density $\hat{\rho}_{\mathrm{f}}$ as shown in Appendix A. It should be noted that the present method uses the LBM for a single-phase fluid with density $\rho_{\mathrm{f}}=1$ without regard to the boundary, since we assume that an incompressible viscous fluid is filled both inside and outside the boundary.

The D2Q9 model has the velocity vectors $\boldsymbol{c}_{i}=(0,0),(0, \pm 1),( \pm 1,0),( \pm 1, \pm 1)$ for $i=1,2, \cdots, 9$. The D3Q15 model has the velocity vectors $\boldsymbol{c}_{i}=(0,0,0)$, $(0,0, \pm 1),(0, \pm 1,0),( \pm 1,0,0),( \pm 1, \pm 1, \pm 1)$ for $i=1,2, \cdots, 15$. The evolution of the particle distribution function $(\mathrm{PDF}) f_{i}(\boldsymbol{x}, t)$ with the velocity $\boldsymbol{c}_{i}$ at the 
point $\boldsymbol{x}$ and time $t$ is computed by the following equations:

$$
f_{i}\left(\boldsymbol{x}+\boldsymbol{c}_{i} \Delta x, t+\Delta t\right)=f_{i}(\boldsymbol{x}, t)-\frac{1}{\tau}\left[f_{i}(\boldsymbol{x}, t)-f_{i}^{\mathrm{eq}}(p(\boldsymbol{x}, t), \boldsymbol{u}(\boldsymbol{x}, t))\right],
$$

where $\Delta x$ is a lattice spacing, $\Delta t$ is the time step during which the particles travel one lattice spacing, $f_{i}^{\text {eq }}$ is an equilibrium $\mathrm{PDF}$, and $\tau$ is a relaxation time of $O(1)$. Note that $\Delta t=S h \Delta x$ where $S h$ is the Strouhal number defined by $S h=\hat{H}_{0} /\left(\hat{t}_{0} \hat{c}\right)=\hat{U}_{0} / \hat{c}=O(\Delta x)$, since $\hat{U}_{0} / \hat{c}$ is of $O(\Delta x)$ in the LBM.

The equilibrium PDF $f_{i}^{\text {eq }}$ of the incompressible model [27] is given by

$$
f_{i}^{\mathrm{eq}}(p, \boldsymbol{u})=E_{i}\left[\frac{p}{c_{\mathrm{s}}^{2}}+\frac{\boldsymbol{c}_{i} \cdot \boldsymbol{u}}{c_{\mathrm{s}}^{2}}+\frac{\left(\boldsymbol{c}_{i} \cdot \boldsymbol{u}\right)^{2}}{2 c_{\mathrm{s}}^{4}}-\frac{\boldsymbol{u} \cdot \boldsymbol{u}}{2 c_{\mathrm{s}}^{2}}\right],
$$

where $E_{1}=4 / 9, E_{2}=\cdots=E_{5}=1 / 9$, and $E_{6}=\cdots=E_{9}=1 / 36$ for the D2Q9 model, $E_{1}=2 / 9, E_{2}=\cdots=E_{7}=1 / 9$, and $E_{8}=\cdots=E_{15}=1 / 72$ for the D3Q15 model, and $c_{\mathrm{s}}$ is the lattice sound speed given by $c_{\mathrm{s}}=1 / \sqrt{3}$. The pressure $p(\boldsymbol{x}, t)$ and the flow velocity $\boldsymbol{u}(\boldsymbol{x}, t)$ are calculated by

$$
\begin{aligned}
& p=c_{\mathrm{s}}^{2} \sum_{i=1}^{b} f_{i}, \\
& \boldsymbol{u}=\sum_{i=1}^{b} f_{i} \boldsymbol{c}_{i},
\end{aligned}
$$

where $b=9$ for the D2Q9 model and $b=15$ for the D3Q15 model. In addition, we can also calculate the stress tensor $\boldsymbol{\sigma}=\left\{\sigma_{\alpha \beta}\right\}(\alpha, \beta=x, y, z)$ as follows:

$$
\sigma_{\alpha \beta}=-\frac{1}{2 \tau} p \delta_{\alpha \beta}-\frac{\tau-1 / 2}{\tau}\left[\sum_{i=1}^{b} f_{i}\left(c_{i \alpha}-u_{\alpha}\right)\left(c_{i \beta}-u_{\beta}\right)-(3 p-1) u_{\alpha} u_{\beta}\right],
$$

where $\delta_{\alpha \beta}$ is the Kronecker delta. Inamuro et al. [25] firstly derived the formulation of the stress tensor $\boldsymbol{\sigma}$ in terms of the PDF instead of the finite difference approximation of the velocity gradient. The above equation (7) is derived on the basis of their work. However, Eq. (7) in the present study is slightly different from Eq. (6) in Ref. [25], since the equilibrium PDF is different. The derivation of Eq. (7) is shown in Appendix B. The kinematic viscosity $\nu$ of the fluid is given by

$$
\nu=c_{\mathrm{s}}^{2}\left(\tau-\frac{1}{2}\right) \Delta x
$$


As described in Ref. [28], it is found that the asymptotic expansions of $p$ and $\boldsymbol{u}$ with respect to $\Delta x$ can be expressed by $p=c_{\mathrm{s}}^{2}+(\Delta x)^{2} p^{(2)}+(\Delta x)^{3} p^{(3)}+$ $(\Delta x)^{4} p^{(4)}+\cdots$ and $\boldsymbol{u}=(\Delta x) \boldsymbol{u}^{(1)}+(\Delta x)^{2} \boldsymbol{u}^{(2)}+(\Delta x)^{3} \boldsymbol{u}^{(3)}+\cdots$, and $p^{(2)}$ 125 and $\boldsymbol{u}^{(1)}$ satisfy the continuity equation and the Navier-Stokes equations for incompressible viscous fluid, while $p^{(3)}$ and $\boldsymbol{u}^{(2)}$ are zero with appropriate initial and boundary conditions [29]. Therefore, the solutions of Eqs. (3)-(6) give the pressure and the fluid velocity for incompressible viscous fluid flows with relative errors of $O\left[(\Delta x)^{2}\right]$ (see Ref. [28]).

When an external body force $\boldsymbol{g}(\boldsymbol{x}, t)$ is applied, the evolution equation (3) of the PDF $f_{i}(\boldsymbol{x}, t)$ can be calculated in a stepwise fashion as follows:

1. $f_{i}(\boldsymbol{x}, t)$ is evolved without the body force by

$$
f_{i}^{*}\left(\boldsymbol{x}+\boldsymbol{c}_{i} \Delta x, t+\Delta t\right)=f_{i}(\boldsymbol{x}, t)-\frac{1}{\tau}\left[f_{i}(\boldsymbol{x}, t)-f_{i}^{\mathrm{eq}}(p(\boldsymbol{x}, t), \boldsymbol{u}(\boldsymbol{x}, t))\right] .
$$

2. $f_{i}^{*}$ is corrected by the body force:

$$
f_{i}(\boldsymbol{x}, t+\Delta t)=f_{i}^{*}(\boldsymbol{x}, t+\Delta t)+\Delta x E_{i} \frac{\boldsymbol{c}_{i} \cdot \boldsymbol{g}(\boldsymbol{x}, t+\Delta t)}{c_{\mathrm{s}}^{2}} .
$$

It should be noted that there are some kinds of the forcing algorithms, e.g., the widely-used one proposed by Guo et al. [30] and the simplest one presented by He et al. [31]. The present forcing algorithm is different not only from the widely-used one but also from the simplest one. In the simplest algorithm, the forcing term is added to the right-hand side of Eq. (3) in the form of $\Delta x E_{i} \boldsymbol{c}_{i}$. $\boldsymbol{g}(\boldsymbol{x}, t) / c_{\mathrm{s}}^{2}$, which means that the second term of the right-hand side of Eq. (10) is $\Delta x E_{i} \boldsymbol{c}_{i} \cdot \boldsymbol{g}\left(\boldsymbol{x}-\boldsymbol{c}_{i} \Delta x, t\right) / c_{\mathrm{s}}^{2}$. Guo et al. [30] proved that the widely-used algorithm is more accurate than the simplest one. The validity of the present forcing algorithm has been confirmed practically in the previous work [26] using a combination of the LBM and the multi-direct forcing method [32] (referred to as MDF-LBM). Also, in our previous works [33, 34] using the MDF-LBM, it has been confirmed that the present forcing algorithm and the widely-used one give almost the same results not only in the level of the total force acting on flapping wings [34] but also in the level of the velocity profile in the laminar boundary 
layer on a flat plate [33], although we have not yet proved the equality or we could not find any proof about it.

\subsection{Calculation of the body force from the discontinuity of the stress tensor}

Supposing that $f_{i}(\boldsymbol{x}, t), p(\boldsymbol{x}, t)$, and $\boldsymbol{u}(\boldsymbol{x}, t)$ are known, the temporary PDF $f_{i}^{*}(\boldsymbol{x}, t+\Delta t)$ is calculated by Eq. (9). Let $\boldsymbol{X}_{k}(t+\Delta t)$ and $\boldsymbol{U}_{k}(t+\Delta t)(k=$ $1,2, \cdots, N)$ be the position of the Lagrangian points of the moving boundary and the boundary velocity at the points, respectively. Suppose that the fluid separated by the boundary be Fluids $\# 1$ and $\# 2$, and that $\boldsymbol{n}_{k}(t+\Delta t)$ be the unit normal vector of the boundary at the Lagrangian point $\boldsymbol{X}_{k}(t+\Delta t)$ pointing to Fluid \#1 (see Fig. 2). Note that the moving boundary is represented by $N$ points, and the boundary Lagrangian points $\boldsymbol{X}_{k}$ generally differ from the background lattice points $\boldsymbol{x}$. Then, the temporary PDF $f_{i}^{*}\left(\boldsymbol{X}_{k}, t+\Delta t\right)$ at a boundary Lagrangian point $\boldsymbol{X}_{k}$ are interpolated by

$$
f_{i}^{*}\left(\boldsymbol{X}_{k}, t+\Delta t\right)=\sum_{\boldsymbol{x}} f_{i}^{*}(\boldsymbol{x}, t+\Delta t) W\left(\boldsymbol{x}-\boldsymbol{X}_{k}\right)(\Delta x)^{d},
$$

where $\sum_{\boldsymbol{x}}$ describes the summation over all lattice points $\boldsymbol{x}, \boldsymbol{d}$ is the dimensionality, and $W$ is a weighting function proposed by Peskin [13] as follows:

$$
\begin{gathered}
W(x, y, z)=\frac{1}{\Delta x} w\left(\frac{x}{\Delta x}\right) \cdot \frac{1}{\Delta x} w\left(\frac{y}{\Delta x}\right) \cdot \frac{1}{\Delta x} w\left(\frac{z}{\Delta x}\right), \\
w(r)= \begin{cases}\frac{1}{8}\left(3-2|r|+\sqrt{1+4|r|-4 r^{2}}\right), & |r| \leq 1, \\
\frac{1}{8}\left(5-2|r|-\sqrt{-7+12|r|-4 r^{2}}\right), & 1 \leq|r| \leq 2, \\
0, & \text { otherwise. }\end{cases}
\end{gathered}
$$

It should be noted that Eq. (12) shows that the three-dimensional weighting function is the product of three one-dimensional weighting functions, and the two-dimensional weighting function can be gained as the product of two onedimensional weighting functions. In many variations of the IBM, the above interpolation procedure has been used for interpolating the fluid velocity. In the present method, we apply the procedure to the interpolation of the PDFs. 


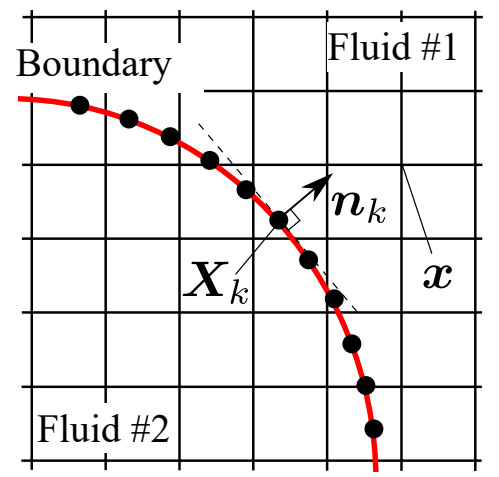

Figure 2: Illustration of boundary Lagrangian points $\boldsymbol{X}_{k}$, the unit normal vector $\boldsymbol{n}_{k}$ at the point, and lattice points $\boldsymbol{x}$.

This process was firstly adopted in the improved momentum exchange-based IB-LBM [21].

The desired PDFs $f_{i}^{[1]}$ and $f_{i}^{[2]}$ which satisfy the no-slip condition on a boundary Lagrangian point $\boldsymbol{X}_{k}$ in Fluids \#1 and \#2, respectively, are obtained by the bounce-back scheme [20] as follows:

$$
\begin{gathered}
f_{i}^{[1]}\left(\boldsymbol{X}_{k}, t+\Delta t\right)= \begin{cases}f_{\bar{i}}^{*}\left(\boldsymbol{X}_{k}, t+\Delta t\right)+2 E_{i} \frac{\boldsymbol{c}_{i} \cdot \boldsymbol{U}_{k}}{c_{\mathrm{s}}^{2}}, & \left(\boldsymbol{n}_{k} \cdot \boldsymbol{c}_{i}>\epsilon\right), \\
f_{i}^{*}\left(\boldsymbol{X}_{k}, t+\Delta t\right), & \text { (otherwise), }\end{cases} \\
f_{i}^{[2]}\left(\boldsymbol{X}_{k}, t+\Delta t\right)= \begin{cases}f_{i}^{*}\left(\boldsymbol{X}_{k}, t+\Delta t\right)+2 E_{i} \frac{\boldsymbol{c}_{i} \cdot \boldsymbol{U}_{k}}{c_{\mathrm{s}}^{2}}, & \left(\boldsymbol{n}_{k} \cdot \boldsymbol{c}_{i}<-\epsilon\right), \\
f_{i}^{*}\left(\boldsymbol{X}_{k}, t+\Delta t\right), & \text { (otherwise), }\end{cases}
\end{gathered}
$$

where $\bar{i}$ is the number which satisfies $\boldsymbol{c}_{\bar{i}}=-\boldsymbol{c}_{i}$, and $\epsilon$ is a small margin value to eliminate the case of $\boldsymbol{n}_{k} \cdot \boldsymbol{c}_{i}=0$. In this study, we set $\epsilon=10^{-14}$. The stress tensors $\boldsymbol{\sigma}^{[1]}\left(\boldsymbol{X}_{k}\right)$ and $\boldsymbol{\sigma}^{[2]}\left(\boldsymbol{X}_{k}\right)$ at a boundary Lagrangian point $\boldsymbol{X}_{k}$ in Fluids $\# 1$ and \#2, respectively, are obtained by Eq. (7) as follows:

$\sigma_{\alpha \beta}^{[\ell]}=-\frac{1}{2 \tau} p^{[\ell]} \delta_{\alpha \beta}-\frac{\tau-1 / 2}{\tau}\left[\sum_{i=1}^{b} f_{i}^{[\ell]}\left(c_{i \alpha}-U_{k \alpha}\right)\left(c_{i \beta}-U_{k \beta}\right)-\left(3 p^{[\ell]}-1\right) U_{k \alpha} U_{k \beta}\right], \quad(\ell=1,2)$,

where $p^{[\ell]}$ is the pressure in Fluid \# $\ell$ calculated from Eq. (5) by using $f_{i}^{[\ell]}$, and $U_{k \alpha}$ is the $\alpha$-component of the velocity $\boldsymbol{U}_{k}$ of a boundary Lagrangian point. It 
should be noted that the calculation of the stress tensor is implemented only on the boundary Lagrangian points. Therefore, the computational overhead for the calculation is not so large.

The body force is determined by the discontinuity of the stress tensor from Eq. (2) as follows:

$$
\boldsymbol{g}\left(\boldsymbol{X}_{k}, t+\Delta t\right) \Delta V=-\left[\boldsymbol{\sigma}^{[1]}\left(\boldsymbol{X}_{k}\right)-\boldsymbol{\sigma}^{[2]}\left(\boldsymbol{X}_{k}\right)\right] \cdot \boldsymbol{n}_{k} \Delta S,
$$

where $\Delta S$ is a small area element given by $S / N$ where $S$ is the area of the body surface. In this method, we choose $N$ so that $\Delta S \lesssim(\Delta x)^{d-1}$. In addition, the body force is not added to one boundary Lagrangian point but a small volume element whose volume is described as $\Delta V$. In this method, $\Delta V$ is taken as $\Delta S \times \Delta x$.

The body force is distributed on lattice points around the boundary as follows:

$$
\boldsymbol{g}(\boldsymbol{x}, t+\Delta t)=\sum_{k=1}^{N} \boldsymbol{g}\left(\boldsymbol{X}_{k}, t+\Delta t\right) W\left(\boldsymbol{x}-\boldsymbol{X}_{k}\right) \Delta V
$$

where the same weighting function (12) as that in the interpolation (11) is used . Finally, the PDF is corrected by the body force in Eq. (10).

\subsection{Force and torque acting on the boundary from the external fluid}

Supposing that Fluid \#1 is the external fluid outside the boundary, the stress $\boldsymbol{P}\left(\boldsymbol{X}_{k}\right)$ acting on the boundary from the external fluid around a Lagrangian point $\boldsymbol{X}_{k}$ is calculated by

$$
\boldsymbol{P}\left(\boldsymbol{X}_{k}\right)=\boldsymbol{\sigma}^{[1]}\left(\boldsymbol{X}_{k}\right) \cdot \boldsymbol{n}_{k}
$$

Therefore, the total force acting on the boundary from the external fluid is given as follows:

$$
\boldsymbol{F}=\sum_{k=1}^{N} \boldsymbol{P}\left(\boldsymbol{X}_{k}\right) \Delta S
$$

In addition, the total torque around a point $\boldsymbol{X}_{\mathrm{c}}$ (e.g., the center of mass of a body) acting on the boundary from the external fluid is given as follows:

$$
\boldsymbol{T}=\sum_{k=1}^{N}\left(\boldsymbol{X}_{k}-\boldsymbol{X}_{\mathrm{c}}\right) \times \boldsymbol{P}\left(\boldsymbol{X}_{k}\right) \Delta S
$$




\subsection{Calculation of the body motion and the boundary Lagrangian points}

In simulations of flows with a freely moving body such as particulate flows, we have to calculate the equation of the body motion with the fluid force (20) and torque (21). In the present study, we consider a rigid body, and its motion is governed by the Newton-Euler equations. In the following, we formulate the same way as the previous work [26].

We consider the motion of the rigid body with density $\rho_{\mathrm{b}}$. Let the inertial reference frame be $\Sigma_{\mathrm{A}}$, and the reference frame fixed to the body be $\Sigma_{\mathrm{B}}$. The origin of $\Sigma_{\mathrm{B}}$ is placed at the center of mass of the rigid body $\boldsymbol{X}_{\mathrm{c}}$. In the following, vectors observed from $\Sigma_{\mathrm{B}}$ have a pre-superscript $\mathrm{B}$, while vectors observed from $\Sigma_{\mathrm{A}}$ have no pre-superscript.

Let $\boldsymbol{U}_{\mathrm{c}}(t)$ be the velocity of $\boldsymbol{X}_{\mathrm{c}}(t)$, and ${ }^{\mathrm{B}} \boldsymbol{\Omega}_{\mathrm{c}}(t)$ the angular velocity around $\boldsymbol{X}_{\mathrm{c}}(t)$. Supposing that we obtain the force $\boldsymbol{F}(t)$ and the torque $\boldsymbol{T}(t)$ acting on the body at time $t$, the motion of the body is governed by the Newton-Euler equations as below:

$$
\begin{aligned}
& M S h \frac{d \boldsymbol{U}_{\mathrm{c}}}{d t}=\boldsymbol{F}, \\
& \boldsymbol{I}_{\mathrm{B}} S h \frac{d^{\mathrm{B}} \boldsymbol{\Omega}_{\mathrm{c}}}{d t}+{ }^{\mathrm{B}} \boldsymbol{\Omega}_{\mathrm{c}} \times\left(\boldsymbol{I}_{\mathrm{B}}{ }^{\mathrm{B}} \boldsymbol{\Omega}_{\mathrm{c}}\right)=\boldsymbol{S}^{\mathrm{T}} \boldsymbol{T},
\end{aligned}
$$

where $M$ is the mass of the rigid body, $\boldsymbol{I}_{\mathrm{B}}$ is the inertia matrix in $\Sigma_{\mathrm{B}}$ (that is, $\boldsymbol{I}_{\mathrm{B}}$ is a constant matrix), and $\boldsymbol{S}(t)$ is the rotational matrix which transforms the basis vectors of $\Sigma_{\mathrm{A}}$ to these of $\Sigma_{\mathrm{B}}$ at time $t$. Note that $\boldsymbol{S}^{\mathrm{T}}$ represents the transpose of $\boldsymbol{S}$. It should be noted that the Strouhal number $S h$ in the time derivative terms in the above equations is derived from the definition the nondimensional variables shown in Appendix A.

A unit quaternion $\boldsymbol{Q}=\left(q_{0}, q_{1}, q_{2}, q_{3}\right)^{\mathrm{T}}$ is used to represent $\boldsymbol{S}$ as below:

$$
\boldsymbol{S}=\left[\begin{array}{ccc}
q_{0}^{2}-q_{2}^{2}-q_{3}^{2}+q_{1}^{2} & 2\left(q_{1} q_{2}-q_{0} q_{3}\right) & 2\left(q_{1} q_{3}+q_{0} q_{2}\right) \\
2\left(q_{1} q_{2}+q_{0} q_{3}\right) & q_{0}^{2}-q_{3}^{2}-q_{1}^{2}+q_{2}^{2} & 2\left(q_{2} q_{3}-q_{0} q_{1}\right) \\
2\left(q_{1} q_{3}-q_{0} q_{2}\right) & 2\left(q_{2} q_{3}+q_{0} q_{1}\right) & q_{0}^{2}-q_{1}^{2}-q_{2}^{2}+q_{3}^{2}
\end{array}\right]
$$


where

$$
q_{0}^{2}+q_{1}^{2}+q_{2}^{2}+q_{3}^{2}=1 .
$$

The kinematic equations of the rigid body are as below:

$$
\begin{aligned}
S h \frac{d \boldsymbol{X}_{\mathrm{c}}}{d t} & =\boldsymbol{U}_{\mathrm{c}}(t), \\
S h \frac{d \boldsymbol{Q}}{d t} & =\boldsymbol{A}(t) \boldsymbol{Q}(t),
\end{aligned}
$$

where $\boldsymbol{A}(t)$ is a $4 \times 4$ matrix which is determined by ${ }^{\mathrm{B}} \boldsymbol{\Omega}_{\mathrm{c}}=\left(\omega_{1}, \omega_{2}, \omega_{3}\right)^{\mathrm{T}}$ as below:

$$
\boldsymbol{A}=\frac{1}{2}\left[\begin{array}{cccc}
0 & -\omega_{1} & -\omega_{2} & -\omega_{3} \\
\omega_{1} & 0 & \omega_{3} & -\omega_{2} \\
\omega_{2} & -\omega_{3} & 0 & \omega_{1} \\
\omega_{3} & \omega_{2} & -\omega_{1} & 0
\end{array}\right] .
$$

We use the second-order Adams-Bashforth method for calculating the above Newton-Euler equations (22) and (23) and the kinematic equations (26) and (27) with the same time step as $\Delta t$ for the fluid motion, and we apply a weakcoupling procedure where the body and fluid motions are calculated alternately.

The position $\boldsymbol{X}_{k}$ and velocity $\boldsymbol{U}_{k}$ of a boundary Lagrangian point are calculated by the following equations:

$$
\begin{aligned}
& \boldsymbol{X}_{k}=\boldsymbol{X}_{\mathrm{c}}+\boldsymbol{S}^{\mathrm{B}} \boldsymbol{X}_{k}, \\
& \boldsymbol{U}_{k}=\boldsymbol{U}_{\mathrm{c}}+\boldsymbol{S}\left({ }^{\mathrm{B}} \boldsymbol{\Omega}_{\mathrm{c}} \times{ }^{\mathrm{B}} \boldsymbol{X}_{k}\right),
\end{aligned}
$$

where it should be noted that ${ }^{\mathrm{B}} \boldsymbol{X}_{k}$ is a constant vector (therefore unchanged from the inital time step), since ${ }^{\mathrm{B}} \boldsymbol{X}_{k}$ represents a boundary Lagrangian point observed from $\Sigma_{\mathrm{B}}$.

\subsection{Algorithm of computation}

The algorithm of computation by the present numerical method is summarized as below.

0 . Suppose the initial value of $f_{i}(\boldsymbol{x}, 0)$ and $\boldsymbol{g}(\boldsymbol{x}, 0)$, and compute $\boldsymbol{u}(\boldsymbol{x}, 0)$ and $p(\boldsymbol{x}, 0)$ by Eqs. (5) and (6). Also, assign $\boldsymbol{X}_{\mathrm{c}}(0), \boldsymbol{S}(0), \boldsymbol{U}_{\mathrm{c}}(0)$, and ${ }^{\mathrm{B}} \boldsymbol{\Omega}_{\mathrm{c}}(0)$. 
1. Compute the force $\boldsymbol{F}(t)$ and the torque $\boldsymbol{T}(t)$ by Eqs. (20) and (21).

2. Compute the velocity $\boldsymbol{U}_{\mathrm{c}}(t+\Delta t)$ and the angular velocity ${ }^{\mathrm{B}} \boldsymbol{\Omega}_{\mathrm{c}}(t+\Delta t)$ of the body by applying the second-order Adams-Bashforth method to Eqs. (22) and (23).

3. Compute the position $\boldsymbol{X}_{\mathrm{c}}(t+\Delta t)$ and the quaternion $\boldsymbol{Q}(t+\Delta t)$ of the body by applying the second-order Adams-Bashforth method to Eqs. (26) and (27), and compute the rotational matrix $\boldsymbol{S}(t+\Delta t)$ by Eq. (24). Then, determine the position $\boldsymbol{X}_{k}(t+\Delta t)$ and velocity $\boldsymbol{U}_{k}(t+\Delta t)$ of the boundary Lagrangian points by Eqs. (29) and (30).

4. Compute the temporary PDFs $f_{i}^{*}(\boldsymbol{x}, t+\Delta t)$ by Eq. (9).

5. Interpolate the temporary PDFs $f_{i}^{*}\left(\boldsymbol{X}_{k}, t+\Delta t\right)$ on the boundary Lagrangian points by Eq. (11).

6. Compute the desired PDFs $f_{i}^{[1]}$ and $f_{i}^{[2]}$ by Eqs. (14) and (15).

7. Compute the stress tensors $\boldsymbol{\sigma}^{[1]}\left(\boldsymbol{X}_{k}, t+\Delta t\right)$ and $\boldsymbol{\sigma}^{[2]}\left(\boldsymbol{X}_{k}, t+\Delta t\right)$ by Eq. (16).

8. Compute the body force $\boldsymbol{g}\left(\boldsymbol{X}_{k}, t+\Delta t\right)$ on the boundary Lagrangian points by Eq. (17).

9. Distribute the body force $\boldsymbol{g}(\boldsymbol{x}, t+\Delta t)$ on the lattice points by Eq. (18).

10. Compute $f_{i}(\boldsymbol{x}, t+\Delta t)$ by Eq. (10), and $p(\boldsymbol{x}, t+\Delta t)$ and $\boldsymbol{u}(\boldsymbol{x}, t+\Delta t)$ by Eqs. (5) and (6).

11. Advance one time step and return to 1.

3.6. Advantages of the present method

The advantages of the present method are as follows:

- The boundary is expressed by Lagrangian points whose arrangement can be independent of the background lattice points.

- The stress tensor can be calculated on the boundary Lagrangian points.

- The total force and torque acting on the boundary from the external fluid can be obtained without calculating the internal mass effect. 
Owing to the first advantage, there is no need to search lattice points which are neighboring on the boundary like staircase-like approximation (e.g., $[1,2]$ ) or to search intersection points where the boundary intersects with the grid line like the interpolated bounce-back method $[35,36]$. In addition, the algorithm using the boundary Lagrangian points remains simple independently of the positional relationship between the boundary and the lattice points, even in moving-boundary flows and/or in three dimensions. This advantage makes the algorithm simple and enables us to easily make the code for moving-boundary flows. As for the second advantage, to our best knowledge, the present method is the first one which enables us to calculate the stress tensor on the boundary Lagrangian points in the class of the diffusive interface method, in which the Peskin's regularized delta function is used for interpolating the velocity on and 240 for distributing the body force around the boundary Lagrangian points. In conventional diffusive interface methods including the momentum exchange-based IB-LBM [18], it is difficult to calculate the pressure and the derivatives of the velocity from the data on some points near the boundary, since the boundary is diffusive. Also, it is impossible to divide the body force into the stress tensors of the internal and external fluids. Although in the present study we consider only rigid boundaries, it is expected that the ability to calculate the stress tensor on the boundary Lagrangian points might be beneficial in calculating movingboundary flows with deformable boundaries. As for the third advantage, if total force and torque acting on the boundary are calculated by using the sum of the body force, it has conventionally been needed to consider the internal mass effect, i.e., to calculate the change of linear and angular momentums of the internal fluid $[14,26,37-39]$. The calculation of the internal mass effect is somewhat cumbersome. In the present method, on the other hand, we can obtain the total force and torque acting on the boundary from the external fluid 255 without calculating the internal mass effect thanks to the second advantage. 


\subsection{Similarities and differences between the present method and two related methods}

Here, we discuss similarities and differences between the present method and two related methods, i.e., the interpolated bounce-back method $[35,36]$ and the 
clude procedures to interpolate the PDF, to calculate the desired PDFs by the bounce-back scheme, and to calculate the body force from the desired PDFs. The essential difference between the present method and the momentum exchange-based IB-LBM is in how to calculate the body force from the desired PDFs. In the present method the body force is calculated from the discontinuity of the stress tensor obtained by the desired PDFs, while in the momentum exchange-based IB-LBM the body force is calculated from the amount of the momentum exchange obtained by the desired PDFs. The present method utilizes the calculation of the stress tensor, since it gives the second and third advantages as shown in Section 3.6.

The present method should be classified into the IBM. We consider the key concept of the IBM as imposing the no-slip condition by using the body force, and therefore a method based on the concept should belong to the IBM. One might consider that the interpolation of the velocity is also a key concept of the IBM. Although many variations of the IBM includes the interpolation of the velocity, it is not a key concept for classifying the variations of the IBM in the widely accepted review of the IBM by Mittal and Iaccarino [5]. In addition, the interpolation of the velocity can be replaced by that of the PDF in the LBM, and at least the momentum exchange-based IB-LBM does not include it. Therefore, the interpolation of the velocity is not considered to be an essential procedure in the IBM.

\section{Results}

In this section, we examine the accuracy of the present IB-LBM by applying it to simulations of moving-boundary flows.

\subsection{Taylor-Couette flow}

First, we consider flows between two concentric circular cylinders. Let the axial direction be the $z$-axis and a plane normal to the $z$-axis be the $x$ - $y$ plane. The radius of the inner cylinder is $R_{1}$ and that of the outer cylinder is $R_{2}=2 R_{1}$. 
Supposing that the outer cylinder is fixed, we consider a flow induced by rotating the inner cylinder around the $z$-axis (a Taylor-Couette flow).

At the initial time $t=0$, the fluid is at rest, and then the inner cylinder rotates around the $z$-axis with the following angular speed:

$$
\Omega_{\mathrm{c}}(t)=\frac{1}{2}\left[1+\tanh \left(\frac{U_{\max } t-0.4 R_{1}}{0.1 R_{1}}\right)\right] \frac{U_{\max }}{R_{1}},
$$

where $U_{\max }$ is the maximum rotating speed. It is noted that $\Omega_{\mathrm{c}}(t)$ tends to $U_{\max } / R_{1}$ as $t \rightarrow \infty$. The computational result obtained by the condition (31) can reach its steady value faster than by the condition that the inner cylinder impulsively starts, that is, $\Omega_{\mathrm{c}}(t)=U_{\max } / R_{1}$, but those steady values are coincident. The steady solution for this problem is given by

$$
\begin{aligned}
u_{\theta}(r) & =-\frac{R_{1} U_{\max }}{R_{2}^{2}-R_{1}^{2}}\left[1-\left(\frac{R_{2}}{r}\right)^{2}\right] r, \\
p(r) & =\left(\frac{R_{1} U_{\max }}{R_{2}^{2}-R_{1}^{2}}\right)^{2}\left[\frac{r^{2}}{2}-\frac{R_{2}^{4}}{2 r^{2}}-2 R_{2}^{2} \ln \left(\frac{r}{R_{2}}\right)\right]+p_{0},
\end{aligned}
$$

where $u_{\theta}$ is the azimuthal flow velocity, $r$ is the distance from the center of the cylinder, and $p_{0}$ is a reference pressure. In this simulation, we choose $p_{0}$ so that the calculated and analytical values of $p$ are coincident at the point $(x, y)=\left(\left(R_{1}+R_{2}\right) / 2,0\right)$. The torque acting on the inner cylinder is given by

$$
T_{z}=-4 \pi \nu R_{1} U_{\max } \frac{R_{2}^{2}}{R_{2}^{2}-R_{1}^{2}} .
$$

Since the flow is not induced in the $z$-direction, we use the two dimensional LBM and calculate the flow only in the $x-y$ plane. We take a computational domain of size $[-H, H] \times[-H, H]$ where $H=R_{2}+3 \Delta x$. All sides of the computational domain are periodic. The Reynolds number defined by $R e=U_{\max } R_{2} / \nu$ is fixed to be 10 and the relaxation time is set to $\tau=0.68$ in this simulation. The number of Lagrangian boundary points $N$ is set to be $4\left(2 R_{1}+1\right)$ for the inner cylinder and $4\left(2 R_{2}+1\right)$ for the outer cylinder. The volume element $\Delta V$ at each boundary point depends on $R_{1}$ and $R_{2}$, but is in the range of $0.7(\Delta x)^{2}$ to $3250.8(\Delta x)^{2}$ for both inner and outer cylinders in this calculation. 

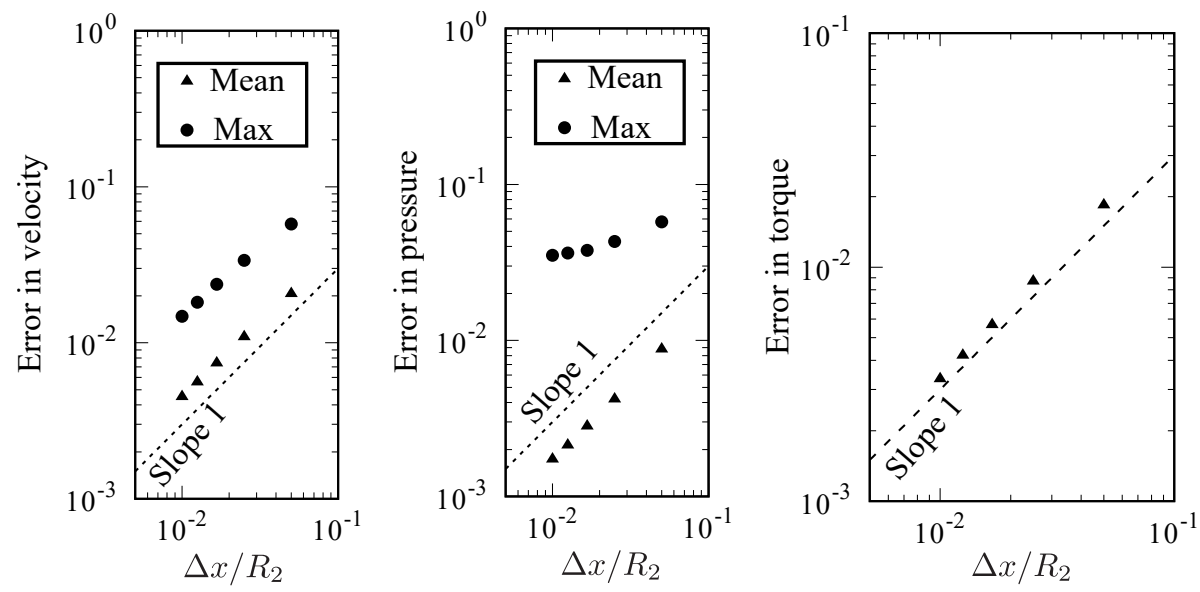

Figure 3: Errors in the velocity, the pressure, and the torque acting on the inner cylinder in the simulation of a Taylor-Couette flow for various spatial resolutions.

We calculate errors from the above analytical solutions in the velocity, pressure, and torque acting on the inner cylinder. The maximum and mean errors in the velocity $\boldsymbol{u}$ are given by

$$
\begin{aligned}
E_{\max }(\boldsymbol{u}) & =\frac{\max \left\{\left\|\boldsymbol{u}_{\text {calc }}-\boldsymbol{u}_{\text {ex }}\right\| ; R_{1}^{2} \leq x^{2}+y^{2} \leq R_{2}^{2}\right\}}{U_{\max }}, \\
E_{\text {mean }}(\boldsymbol{u}) & =\frac{\sum_{R_{1}^{2} \leq x^{2}+y^{2} \leq R_{2}^{2}}\left\|\boldsymbol{u}_{\text {calc }}-\boldsymbol{u}_{\text {ex }}\right\|(\Delta x)^{2}}{\pi\left(R_{2}^{2}-R_{1}^{2}\right) U_{\max }},
\end{aligned}
$$

where $\boldsymbol{u}_{\text {calc }}$ is a calculated value of $\boldsymbol{u}, \boldsymbol{u}_{\text {ex }}$ is the analytical value of $\boldsymbol{u}$, and $\sum_{R_{1}^{2} \leq x^{2}+y^{2} \leq R_{2}^{2}}$ means the summation over lattice points in the range $R_{1}^{2} \leq$ $x^{2}+y^{2} \leq R_{2}^{2}$. The maximum and mean errors in the pressure $p$ are given by

$$
\begin{aligned}
E_{\max }(p) & =\frac{\max \left\{\left|p_{\text {calc }}-p_{\text {ex }}\right| ; R_{1}^{2} \leq x^{2}+y^{2} \leq R_{2}^{2}\right\}}{U_{\max }^{2}}, \\
E_{\text {mean }}(p) & =\frac{\sum_{R_{1}^{2} \leq x^{2}+y^{2} \leq R_{2}^{2}}\left|p_{\text {calc }}-p_{\text {ex }}\right|(\Delta x)^{2}}{\pi\left(R_{2}^{2}-R_{1}^{2}\right) U_{\max }^{2}},
\end{aligned}
$$

where $p_{\text {calc }}$ is a calculated value of $p$, and $p_{\text {ex }}$ is the analytical value of $p$. The error in $T_{z}$ is given by

$$
E\left(T_{z}\right)=\left|\frac{T_{z \mathrm{calc}}-T_{z \mathrm{ex}}}{T_{z \mathrm{ex}}}\right|,
$$

where $T_{z \text { calc }}$ is a calculated value of $T_{z}$, and $T_{z \text { ex }}$ is the analytical value of $T_{z}$. Fig. 3 shows the decay of the errors against the lattice spacing $\Delta x$. We can see 
from this figure that the present results have almost first-order accuracy in the velocity, the pressure, and the torque acting on the inner cylinder. Only the maximum error in the pressure shows a worse convergence rate. This means that the order of accuracy of the LBM, which is formally second-order accurate, decreases by using the IBM. This is because the discontinuities of the pressure and the velocity gradient (i.e., the stress tensor) make a decrease in the order of accuracy $[6,40,41]$. It should be noted that the order of accuracy is comparable with other diffusive IB-LBMs (e.g., [41]).

It is well known that the relaxation time $\tau$ is playing a role in determining the position of the boundary expressed by the bounce-back scheme [42]. Therefore, it is important to check the effect of $\tau$ on the accuracy. Fig. 4 shows $E_{\text {mean }}(\boldsymbol{u})$, $E_{\text {mean }}(p)$, and $E\left(T_{z}\right)$ against $\tau$ in the range of $0.52 \leq \tau \leq 3.0$ for $R_{1}=30 \Delta x$. 340 In this figure, the numerical results by the MDF-LBM [26] are also shown for comparison. We can see from this figure that all kinds of the errors show a similar tendency, i.e., they decrease for $\tau$ smaller than a certain value and increase for $\tau$ larger than the value, although the value of $\tau$ which gives the minimum error depends on the kind of the errors and on the methods. On the whole, the errors obtained by the present method are reasonably small for $0.5<\tau \lesssim 1.0$, and smaller than those by MDF-LBM in this range of $\tau$. Therefore, it is recommended that the present method should be used for $0.5<\tau \lesssim 1.0$.

\subsection{Translationally oscillating circular cylinder in a stationary fluid}

Next, we consider an oscillating circular cylinder in a stationary fluid. This problem was studied both experimentally and numerically by Dütsch et al. [43]. The diameter of the cylinder is $D$, and the width and the height of the computational domain are $55 D$ and $35 D$, respectively. The fluid initially at rest, and the cylinder placed at the center of the domain suddenly starts to oscillate with 

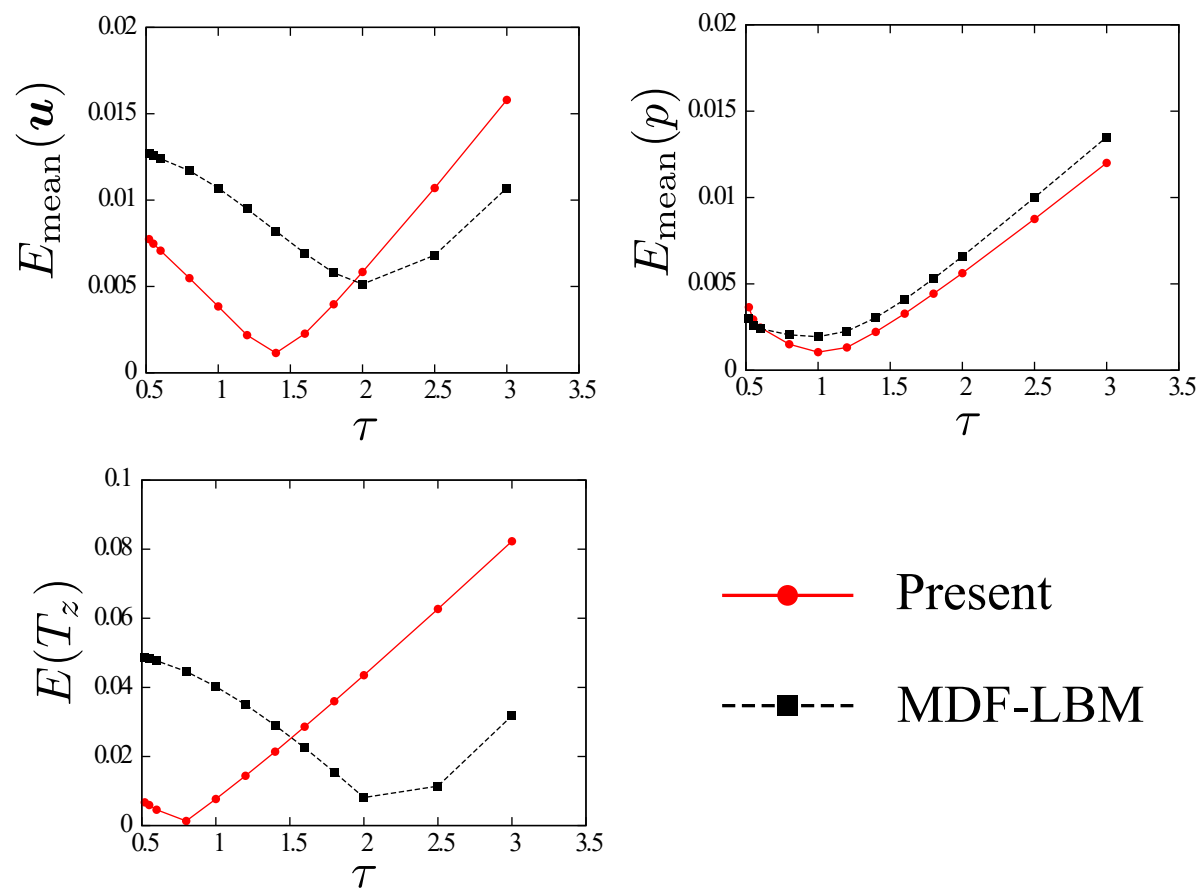

Figure 4: Errors in the velocity, the pressure, and the torque acting on the inner cylinder in the simulation of a Taylor-Couette flow with $R_{1}=30 \Delta x$ for various relaxation times $\tau$ compared with the numerical results by the MDF-LBM [26].

the following speed:

$$
\begin{aligned}
& U_{\mathrm{c}}(t)=-U_{\max } \cos \left(\frac{2 \pi}{T} t\right), \\
& V_{\mathrm{c}}(t)=0
\end{aligned}
$$

where $U_{\mathrm{c}}(t)$ and $V_{\mathrm{c}}(t)$ are the respective velocity components in the $x$ - and $y$ directions of the cylinder, $U_{\max }$ is the amplitude of the velocity, and $T$ is the period. In the simulation, the diameter of the circular cylinder is $D=50 \Delta x$, and the number of boundary Lagrangian points along the cylinder surface is $N=204$. The Neumann boundary condition is used at the outer boundary of the domain, i.e., $\boldsymbol{n}_{\mathrm{d}} \cdot \nabla f_{i}=0$ (where $\boldsymbol{n}_{\mathrm{d}}$ is the unit vector normal to the boundary). The governing parameters of the system are the Reynolds number $R e=U_{\max } D / \nu$ and the Keulegan-Carpenter number $K C=2 \pi A_{\mathrm{m}} / D$ where 


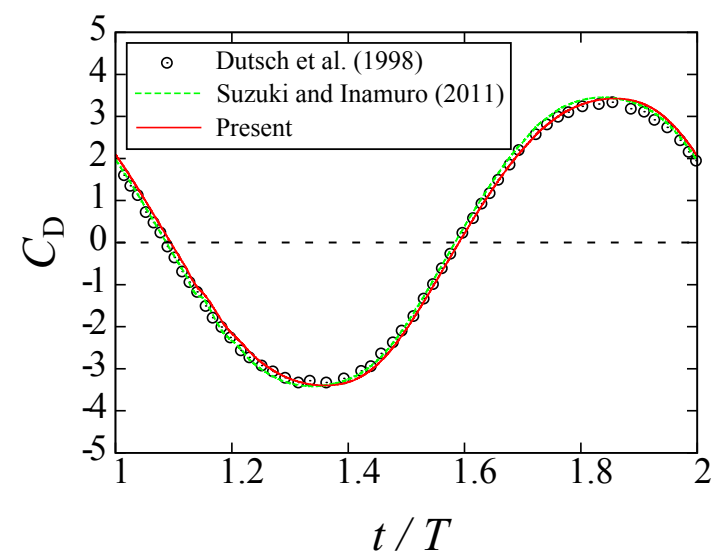

Figure 5: Time variation of the drag coefficient of a translationally oscillating circular cylinder for $R e=100$ with $K C=5$. The result obtained by the present method is compared with the numerical results by Dütsch et al. [43] and with those by Suzuki and Inamuro [26] using the Lagrangian points approximation.

$A_{\mathrm{m}}$ is the spatial amplitude of the oscillation. We calculate a case for $R e=100$ with $K C=5$. In this simulation, we set $U_{\max }=0.03$ and $\tau=0.5450$.

Fig. 5 shows the time variation of the drag coefficient $C_{\mathrm{D}}=2 F_{x} /\left(\rho_{\mathrm{f}} U_{\max }^{2} D\right)$ where $F_{x}$ is the force acting on the body in the $x$-direction. It should be noted that since the results by Dütsch et al. [43] are for periods after initial transient flows, we cut off the transients for the first period $(0 \leq t / T<1)$ of our results. Hence, the data in Fig. 5 begin with the second period. It is seen from Fig. 5 that the present result quantitatively agrees with the numerical results by Dütsch et al. [43] and with those by Suzuki and Inamuro [26] using the Lagrangian points approximation. In addition, we can see that there is no spurious oscillation in the time variation of $C_{\mathrm{D}}$ of the present result. This result means that the present method can give an accurate force acting on a moving body.

\subsection{The sedimentation of an elliptical cylinder}

Thirdly, we consider an elliptical cylinder falling under gravity in a closed narrow domain filled with fluid. The computational condition and the coordinate system are shown in Fig. 6. The major and minor axes of the elliptical 


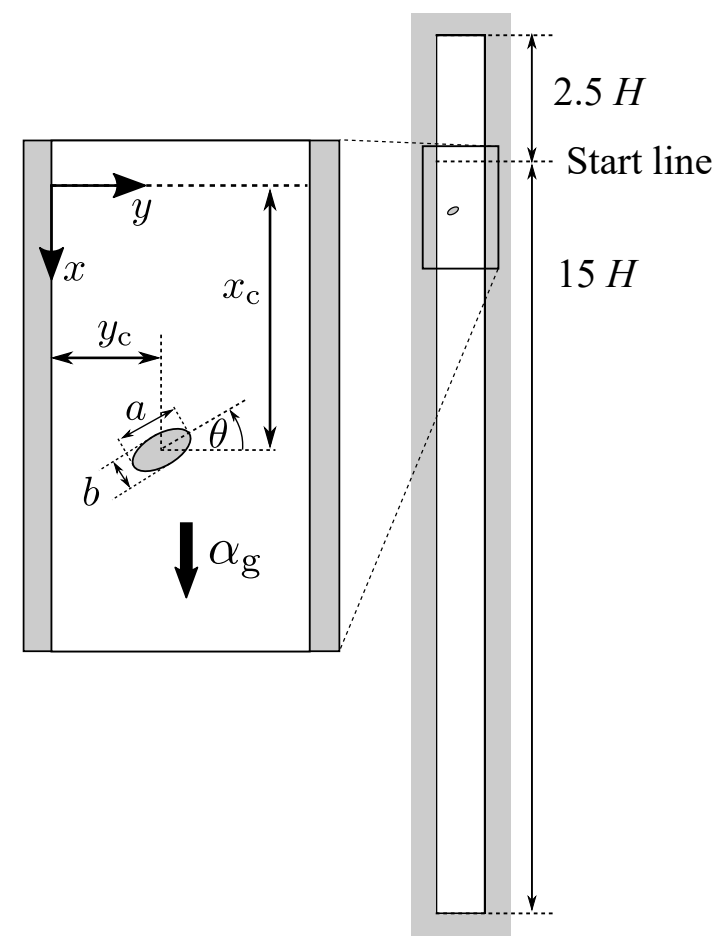

Figure 6: The computational domain for an elliptical cylinder falling in a closed narrow domain. The elliptical cylinder is driven by a constant gravity acceleration $\alpha_{\mathrm{g}}$. The coordinate $x$ is taken in the direction of the gravity and $y$ is in the width of the channel. $\theta$ is the cylinder's angle of inclination with respect to the $y$-axis.

cylinder are $a$ and $b$, respectively. The width of the domain is $H$ and the height of the domain is $17.5 H$. The major axis is $a=H / 4$ and the minor axis is $b=H / 8$. The gravity acceleration is $\alpha_{\mathrm{g}}$. The density of the elliptical cylinder is $\rho_{\mathrm{b}}$. It should be noted that the density of both the fluids inside and outside the body is the same, and $\rho_{\mathrm{b}}$ is used only for calculating the equations of the body motion (22) and (23). Letting the density ratio of the elliptical cylinder to the fluid be $\gamma=\rho_{\mathrm{b}} / \rho_{\mathrm{f}}$, the mass and the inertia moment of the elliptical cylinder are $M=\rho_{\mathrm{b}}(\pi a b / 4)$ and $I_{\mathrm{B}}=M\left(a^{2}+b^{2}\right) / 16$, respectively. The net gravitational force is $F_{\mathrm{g}}=(1-1 / \gamma) M \alpha_{\mathrm{g}}$. In the simulation, we set $H=200 \Delta x$. The number of boundary Lagrangian points is $N=154$. The fluid is initially at rest, and the elliptical cylinder starts its motion at the initial position of $\left(x_{\mathrm{c}}, y_{\mathrm{c}}\right)=(0,0.5 \mathrm{H})$ 
Table 1: The parameters used and obtained in simulations of the sedimentation of an elliptical cylinder.

\begin{tabular}{lllll}
\hline$\gamma$ & $\tau$ & $\alpha_{\mathrm{g}} \Delta x$ & $u_{\mathrm{t}}$ & $R e$ \\
\hline 1.1 & 0.6364 & $1.620 \times 10^{-4}$ & 0.0115 & 12.6 \\
1.5 & 0.6364 & $1.620 \times 10^{-4}$ & 0.0305 & 33.5 \\
\hline
\end{tabular}

385 with fluid. This problem was measured using a PIV system by ten Cate et al. [37]. The experimental conditions are presented below with dimensional variables. The dimensions of the box are depth $\times$ width $\times$ height $=100 \times$ $100 \times 160 \mathrm{~mm}$. The diameter of the sphere is $\hat{D}_{\mathrm{p}}=15 \mathrm{~mm}$ and its density is ${ }_{405} \hat{\rho}_{\mathrm{b}}=1120 \mathrm{~kg} / \mathrm{m}^{3}$. The fluid is initially at rest, and the sphere starts its motion at a height $120 \mathrm{~mm}$ from the bottom of the domain due to the gravity acceleration $\hat{\alpha}_{\mathrm{g}}=9.8 \mathrm{~m} / \mathrm{s}^{2}$. In the simulation, the domain is divided into $200 \times 200 \times 320$ 

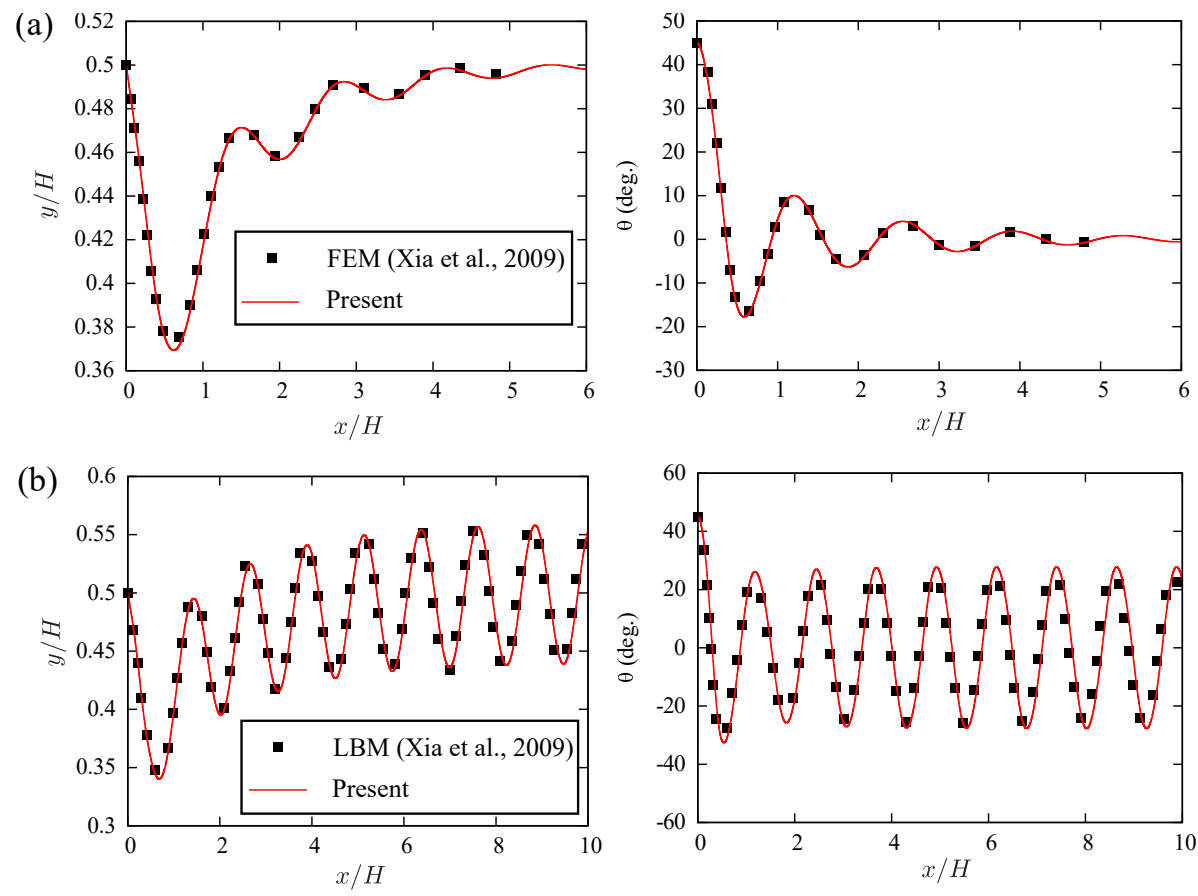

Figure 7: Trajectory and orientation of the elliptical cylinder for $(\mathrm{a})(\gamma, R e)=(1.1,12.6)$ and (b) $(\gamma, R e)=(1.5,33.5)$. The left figures show the horizontal position of the center of the elliptical cylinder, and the right figures show the orientation of the elliptical cylinder. The present results are compared with the numerical results by Xia et al. [44].

lattice grid. Hence, 30 lattice spacings are used for the diameter of the sphere. The boundary Lagrangian points on the sphere should be arranged uniformly and symmetrically. In the present study, we use a spherical geodesic grid with refinement loop $n=5$ [45]. The number of the boundary Lagrangian points is $N=10242$. The bounce-back scheme [20] is used for enforcing the no-slip condition on the outer boundary of the box. In the experiments by ten Cate et al. [37], a liquid free surface exists at the top of the domain. In spite of this, we use the no-slip condition at the top of the domain instead of the free surface condition. This is because it is difficult to achieve the free surface condition, and the influence of the mismatch of the boundary conditions on the top of the domain is considered to be small. In addition, several researchers also use the 
no-slip condition and obtain good results in the same problem $[38,46,47]$. We use no repulsive force when the gap $L$ between the sphere and the bottom of the domain is close to zero, while Feng et al. $[38,46]$ employed in their simulations. Hence, we stop our simulations when $L$ is less than 3 lattice spacings. The governing parameters of the system are the density ratio of the sphere to the fluid $\gamma=\hat{\rho}_{\mathrm{b}} / \hat{\rho}_{\mathrm{f}}$ and the Reynolds number $R e=\hat{\rho}_{\mathrm{f}} \hat{u}_{\infty} \hat{D}_{\mathrm{p}} / \hat{\mu}$, where $\hat{u}_{\infty}$ is the sedimentation velocity of a sphere in an infinite domain [37] and $\hat{\mu}$ is the fluid viscosity. It is pointed out that the effective radius of the sphere is slightly bigger than the input radius in the conventional IB-LBM simulations [37, 38]. ten Cate et al. [37] calculated the effective radius by a calibration procedure, and used a length scale based on the effective radius. Feng and Michaelides [38] distributed the boundary points on a spherical surface whose radius is slightly smaller than that of the sphere. In this simulation, however, we do not use the effective radius.

We calculate four cases as listed in Table 2. The simulated results of the gap $L$ and the falling velocity $u$ are shown in Fig. 8 with the experimental results by ten Cate et al. [37]. We can see from this figure that the present results have a good agreement with the experimental results by ten Cate et al. [37] for all four cases. This result means that the present method can be successfully applied to three-dimensional moving-boundary flows.

We mentioned that in the present method the effective radius is not used,

Table 2: Fluid properties in the experiment by ten Cate et al. [37] and the parameters used for simulations of the sedimentation of a sphere.

\begin{tabular}{rllll}
\hline$R e$ & $\hat{\rho}_{\mathrm{f}}\left(\mathrm{kg} / \mathrm{m}^{3}\right)$ & $\hat{\mu}\left(\times 10^{-3} \mathrm{Ns} / \mathrm{m}^{2}\right)$ & $\tau$ & $\alpha_{\mathrm{g}} \Delta x$ \\
\hline 1.5 & 970 & 373 & 0.8000 & $8.284 \times 10^{-5}$ \\
4.1 & 965 & 212 & 0.8000 & $2.538 \times 10^{-4}$ \\
11.6 & 962 & 113 & 0.7328 & $5.344 \times 10^{-4}$ \\
32.2 & 960 & 58 & 0.5839 & $2.622 \times 10^{-4}$ \\
\hline
\end{tabular}



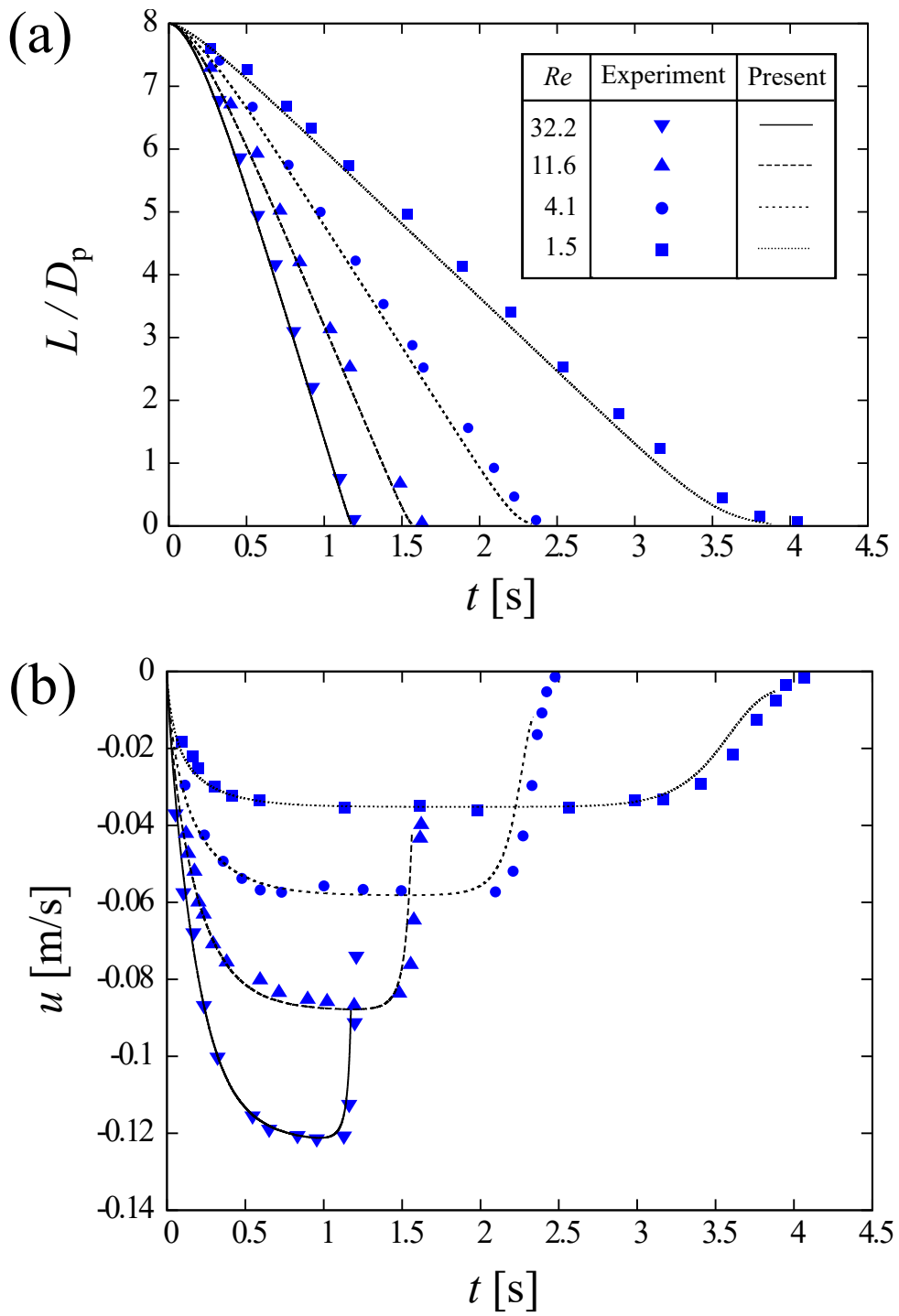

Figure 8: Comparisons of results by the present method to experimental results by ten Cate et al. [37]. (a) The time variation of the gap $L$ between the sphere and the bottom of the domain and (b) the time variation of the velocity of the sphere $u$.

while in other IB-LBMs [37, 38] some calibrations considering the effective radius were implemented. On the other hand, the effective position of the boundary expressed by the bounce-back scheme is affected by the relaxation time $\tau$ [42]. 
Therefore, one might consider that the present result is effectively calibrated by changing $\tau$, rather than the lack of the effective radius. In order to examine the effect of $\tau$ and compare another IB-LBM which does not use the bounce-back scheme, we calculate this problem for $\tau=0.52,0.58$, and 0.80 , and compare the results of the present method with those of the MDF-LBM [26]. It should be noted that the MDF-LBM does not use the bounce-back scheme, and any calibration is not implemented in the simulation by this method. Fig. 9 shows the time variation of the velocity of the sphere at $R e=32.2$. We can see from this figure that the results of the MDF-LBM overlap each other, and the results of the present method have very small deviations by changing $\tau$. In addition, the difference between the results of the present method and those of the MDFLBM is larger than the deviations by changing $\tau$, and the results of the present method are closer to the experimental results than those of the MDF-LBM. The difference between the results of the MDF-LBM and the experimental result is considered to be the effect of the effective radius. Therefore, we can conclude that the present method can give a good result without any calibration with respect to $\tau$ and the effective radius.

\section{Discussions}

As shown in the previous section, the present method can give accurate results in moving-boundary flows. However, it still has some problems in other points of view. In this section, we discuss the problems of the present method and a possible remedy for them.

\subsection{Penetration}

In the present method, the no-slip condition on the boundary is not imposed directly unlike the velocity-based IB-LBM such as the implicit velocity correction-based IB-LBM [48] and the MDF-LBM [26]. This might lead penetration of the fluid across the boundary.

470

In order to check such penetration, we consider a well-known benchmark problem of the flow over a circular cylinder. The diameter of the circular cylinder 


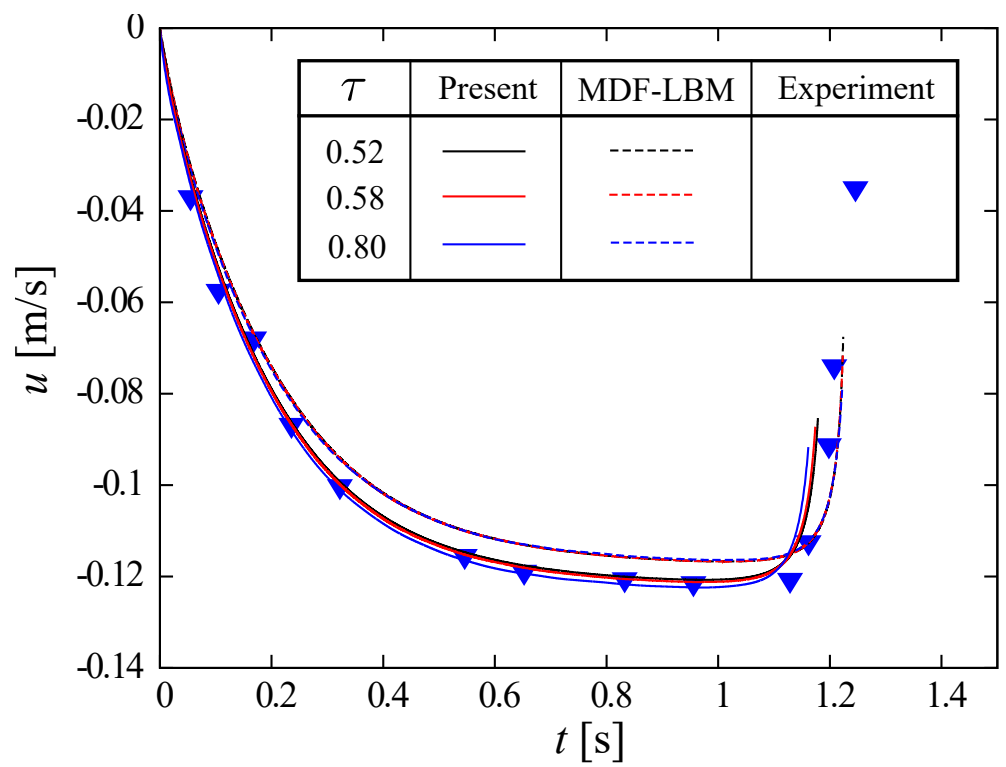

Figure 9: Time variation of the velocity of the sphere $u$ at $R e=32.2$ for various relaxation times $\tau$ compared with the numerical results by the MDF-LBM [26] and the experimental results by ten Cate et al. [37].

is $D_{\mathrm{s}}$. The computational domain is $\left[-16 D_{\mathrm{s}}, 24 D_{\mathrm{s}}\right] \times\left[-20 D_{\mathrm{s}}, 20 D_{\mathrm{s}}\right]$. The center of the circular cylinder is located at $(x, y)=(0,0)$. The pressure and velocity on all of the boundaries of the computational domain are imposed as $p=p_{\infty}$ and $\boldsymbol{u}=\left(u_{\infty}, 0\right)$, where $p_{\infty}$ and $u_{\infty}$ are constant. We assume the unknown PDFs on the boundaries are equal to the equilibrium PDFs with the pressure and velocity on the boundaries. The pressure and velocity are initially set to be uniform with the same values as those of the boundary condition. The governing parameter of the system is the Reynolds number defined by $R e=u_{\infty} D_{\mathrm{s}} / \nu$. In this simulation, we set $D_{\mathrm{s}}=50 \Delta x, N=204, p_{\infty}=1 / 3$, and $u_{\infty}=0.03$.

Fig. 10(a) shows the streamlines around the circular cylinder at $R e=40$ when the flow reaches the steady state. We can see from this figure that penetration occurs across the boundary. This is because the present method does not impose the no-slip condition directly. Especially when the pressure discontinuity across the boundary is very large like this problem, the embankment of 
(a)

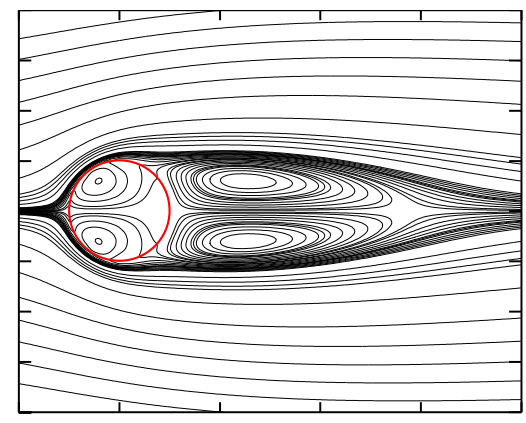

(b)

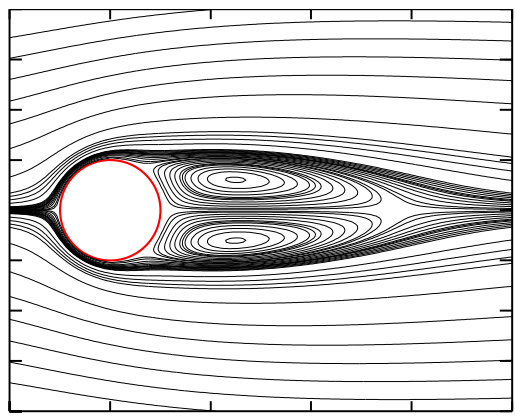

Figure 10: Streamlines in the flow past a circular cylinder at $R e=40$ for (a) the present method and (b) the present method with preprocessing.

the body force might be broken due to the large pressure gradient. Actually, the streamline penetrates around the stagnation point in the front of the circular cylinder. Table 3 shows the drag coefficient defined by $C_{\mathrm{D}}=2 F_{x} /\left(\rho_{\mathrm{f}} u_{\infty}^{2} D_{\mathrm{s}}\right)$ and the wake length $L_{\mathrm{w}} / D_{\mathrm{s}}$ (where $L_{\mathrm{w}}$ is the length of the recirculation region from the rearmost point of the cylinder to the end of the wake) with other experimental $[49,50]$ and numerical $[48,51-53]$ results for $R e=20$ and 40 . It can be seen from this table that the present results have a good agreement with the other results. This suggests that the penetration has a small effect on the net force and the external flow field at small Reynolds numbers.

Table 4 shows the results for $R e=200$, in which the vortex shedding occurs, i.e., the drag and lift forces oscillate periodically. This table contains the drag coefficient defined as above, the lift coefficient defined by $C_{\mathrm{L}}=2 F_{y} /\left(\rho_{\mathrm{f}} u_{\infty}^{2} D_{\mathrm{s}}\right)$, and the Strouhal number $S t=f_{\mathrm{s}} D_{\mathrm{s}} / u_{\infty}$ of the vortex shedding (where $f_{\mathrm{s}}$ is the shedding frequency) with other experimental [54] and numerical [53, 55-57] results. It can be seen from this table that the present method slightly overestimates the drag and lift coefficients compared with other numerical results, while the present result of the Strouhal number of the vortex shedding has a good agreement. This overestimation of the force acting on the boundary might be an effect of the penetration.

As shown above, the penetration might have harmful effects for a higher Reynolds number due to unsteadiness and complexity of the flow. In general, 
Table 3: Comparison of the drag coefficient $C_{\mathrm{D}}$ and the wake length $L_{\mathrm{w}} / D_{\mathrm{s}}$ at $R e=20$ and 40.

\begin{tabular}{llll}
\hline$R e$ & References & $C_{\mathrm{D}}$ & $L_{\mathrm{w}} / D_{\mathrm{s}}$ \\
\hline 20 & Tritton [49] (Exp.) & 2.09 & - \\
& Coutanceau and Bouard [50] (Exp.) & - & 0.93 \\
Dennis and Cheng [51] & 2.045 & 0.94 \\
He and Doolen [52] & 2.152 & 0.921 \\
Taira and Colonius [53] & 2.07 & 0.94 \\
Wu and Shu [48] & 2.091 & 0.93 \\
Present & 2.091 & 0.94 \\
Present (preprocessing) & 2.070 & 0.96 \\
\hline T0itton [49] (Exp.) & 1.59 & - \\
Coutanceau and Bouard [50] (Exp.) & - & 2.13 \\
Dennis and Cheng [51] & 1.522 & 2.35 \\
He and Doolen [52] & 1.550 & 2.25 \\
Taira and Colonius [53] & 1.54 & 2.30 \\
Wu and Shu [48] & 1.565 & 2.31 \\
Present & 1.568 & 2.36 \\
Present (preprocessing) & 1.548 & 2.34 \\
\hline
\end{tabular}

there are two ways to prevent the penetration. The first way is to enforce the no-slip condition on the boundary directly and accurately like the implicit velocity correction method [48] and the multi-direct forcing method [32]. The second way is to enforce the velocity of a rigid body on the internal fluid inside the boundary like the smoothed-profile method (SPM) $[58,59]$. In this study, we consider a simple preprocessing in the second way. It should be noted that the preprocessing considered here can be applied only to a circular cylinder and a sphere. As for more complicated and/or deformable bodies, the extension of 515 this method remains in future work. 
Table 4: Comparison of the drag coefficient $C_{\mathrm{D}}$, the lift coefficient $C_{\mathrm{L}}$, and the Strouhal number $S t$ of the vortex shedding at $R e=200$.

\begin{tabular}{llll}
\hline References & $C_{\mathrm{D}}$ & $C_{\mathrm{L}}$ & $S t$ \\
\hline Roshko [54] (Exp.) & - & - & 0.19 \\
Liu et al. [55] & $1.31 \pm 0.049$ & \pm 0.69 & 0.192 \\
Linnick and Fasel [56] & $1.34 \pm 0.044$ & \pm 0.69 & 0.197 \\
Taira and Colonius [53] & $1.35 \pm 0.048$ & \pm 0.68 & 0.196 \\
Wang et al. [57] & $1.43 \pm 0.051$ & \pm 0.75 & 0.195 \\
Present & $1.46 \pm 0.050$ & \pm 0.74 & 0.196 \\
Present (preprocessing) & $1.39 \pm 0.049$ & \pm 0.72 & 0.194 \\
\hline
\end{tabular}

In the preprocessing for a circular cylinder or a sphere whose center is located at $X_{\mathrm{c}}$, we use the following smoothed profile:

$$
\phi(\boldsymbol{x})=s\left(R-\left|\boldsymbol{x}-\boldsymbol{X}_{\mathrm{c}}\right|\right),
$$

where $\boldsymbol{x}$ is a lattice point, $R$ is a radius of the area where the preprocessing is applied, and $s$ is the following function:

$$
s(r)= \begin{cases}0, & (r<-\xi / 2), \\ \frac{1}{2}\left[\sin \left(\frac{\pi r}{\xi}\right)+1\right], & (|r| \leq \xi / 2), \\ 1, & (r>\xi / 2)\end{cases}
$$

where $\xi$ is a thickness of the interface of the profile. In this study, the parameters $R$ and $\xi$ are set to $R=0.5 D_{\mathrm{s}}-0.5 \Delta x$ and $\xi=\Delta x$, respectively, so that the area where the preprocessing is applied does not interfere the calculation of the body force on the boundary points. According to the algorithm presented by Mino et al. [60], the preprocessing body force is calculated as follows:

$$
\boldsymbol{g}_{\mathrm{p}}(\boldsymbol{x}, t+\Delta t)=\phi(\boldsymbol{x}, t+\Delta t) \frac{S h}{\Delta t}\left[\boldsymbol{U}_{\mathrm{in}}(\boldsymbol{x}, t+\Delta t)-\boldsymbol{u}^{*}(\boldsymbol{x}, t+\Delta t)\right],
$$

where $\boldsymbol{U}_{\text {in }}(\boldsymbol{x})$ is the velocity of the body at a lattice point $\boldsymbol{x}$ inside the boundary (e.g., $\boldsymbol{U}_{\text {in }}(\boldsymbol{x})=\mathbf{0}$ for a stationary body), and $\boldsymbol{u}^{*}(\boldsymbol{x}, t+\Delta t)$ is the temporary 


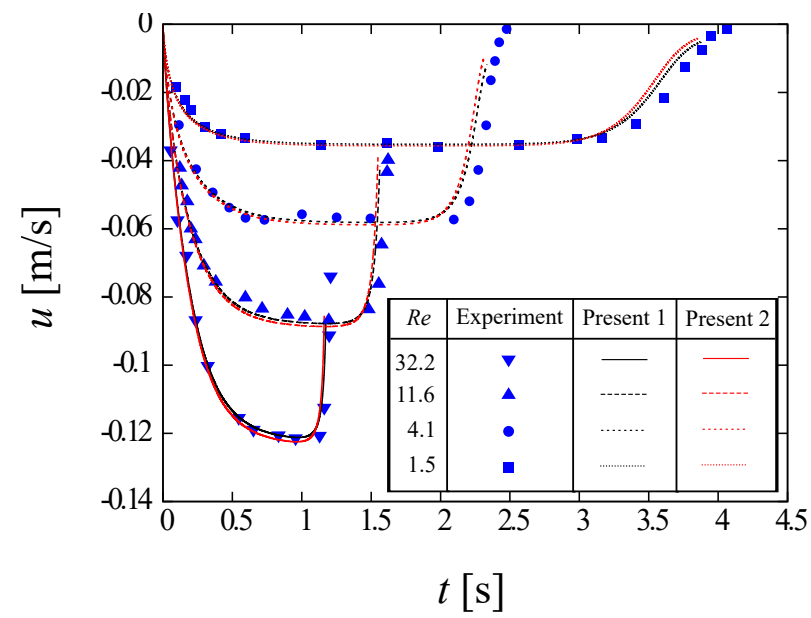

Figure 11: Comparisons of the time variation of the velocity of the sphere $u$ obtained by the present method without/with preprocessing to experimental results by ten Cate et al. [37]. In this figure, 'Present 1' means the present method without preprocessing and 'Present 2' means that with preprocessing.

fluid velocity calculated from Eq. (6) by using the temporary PDF $f_{i}^{*}(\boldsymbol{x}, t+$ $\Delta t$ ) (see Section 3.2), and $S h$ is the Strouhal number described in Section 3.1. Finally, the temporary PDF is corrected by $\boldsymbol{g}_{\mathrm{p}}(\boldsymbol{x}, t+\Delta t)$ in Eq. (10). After the preprocessing, we start the algorithm shown in Section 3.2. It should be noted that the preprocessing body force $\boldsymbol{g}_{\mathrm{p}}$ is not used in calculating the total force and torque acting on the boundary.

Fig. 10(b) shows the streamlines for the present method with the preprocessing. We can see from this figure that the penetration does not occur across the boundary. In addition, it can be seen from Table 3 that the drag coefficient and the wake length obtained by the present method with the preprocessing still have a good agreement with other results, and from Table 4 that the overestimation in the drag and lift coefficients decreases compared with the case without the preprocessing. These results mean that the present preprocessing is effective for preventing the penetration.

The present preprocessing can be applied to moving-boundary flows. Actually, the present method with the preprocessing still gives good results in the 
translationally oscillating circular cylinder in a stationary fluid (Section 4.2) and the sedimentation of a sphere (Section 4.4). For example, the results in the sedimentation of a sphere are shown in Fig. 11. We can see from this figure that even in a three-dimensional moving-boundary flow, the present method with preprocessing gives comparable results with the experimental results.

\subsection{Spurious oscillation of local force}

In the same problem as the previous section, we calculate the pressure coefficient at a boundary Lagrangian point with the argument $\theta$ defined by

$$
C_{p}(\theta)=\frac{p^{[1]}(\theta)-p_{\infty}}{0.5 \rho_{\mathrm{f}} u_{\infty}^{2}}
$$

${ }_{540}$ where $p^{[1]}$ is the pressure on the boundary point calculated by $f_{i}^{[1]}$ (see Section 3.2), supposing that Fluid \#1 is the external fluid. Fig. 12 shows the pressure coefficients against $\theta$ at $R e=40$ obtained by the present method without/with preprocessing and other numerical results [52, 61]. We can see from this figure that the present result without preprocessing has a large error from other results around the stagnation point in the front of the circular cylinder $\left(\theta=0^{\circ}\right)$, and spurious oscillation is observed around $\theta=45^{\circ}$ and $90^{\circ}$. The error around $\theta=0^{\circ}$ should be attributed to the penetration discussed in the previous section. Actually, the error is eliminated in the result with preprocessing. On the other hand, the spurious oscillation around $\theta=45^{\circ}$ and $90^{\circ}$ remains even in the result with preprocessing. This means that the cause of the spurious oscillation is not the penetration. Except the spurious oscillation, the result with preprocessing has a good agreement with other numerical results [52, 61].

It should be noted that we cannot observe significant effects of the spurious oscillation on the total force (see Tables 3 and 4) and on the time variation of the total force (see Fig. 5). The oscillation occurs even when we use not only a larger number of the boundary points but also a finer spatial resolution. In addition, even when the center of the circluar cylinder is slightly deviated from a lattice point, e.g., $(x, y)=(0.5 \Delta x, 0.25 \Delta x)$, the pressure profile is not changed and this oscillation occurs around the same points $\left(\theta=45^{\circ}\right.$ and $\left.90^{\circ}\right)$. This 


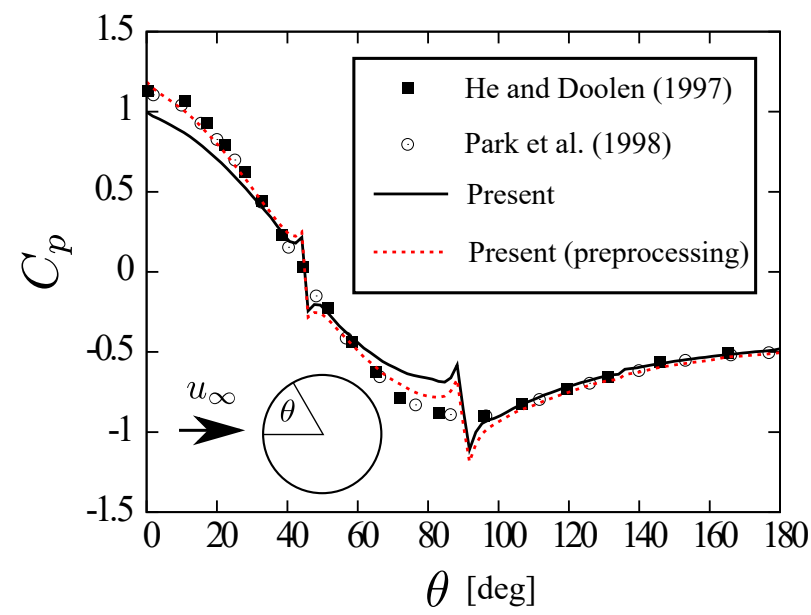

Figure 12: Pressure distributions on the circular cylinder at $R e=40$ obtained by He and Doolen [52], Park et al. [61], and the present method without/with preprocessing.

means that it is independent of the positional relation between the boundary points and the lattice points. Although we cannot completely explain the root cause of the spurious oscillation yet, similar oscillations have been reported in several studies of the LBM with the interpolated bounce-back scheme [62] and the velocity-based IBM with other flow solver [63]. Therefore, the cause of the spurious oscillation has to be investigated from both viewpoints of the LBM and the IBM. In particular, the recent study by Goza et al. [63] might give a potential hint for solving this problem. In their study using the velocity-based IBM with a finite-volume method for the Navier-Stokes equations, the source of the spurious oscillation is attributed to the fact that the equation for the body force is an ill-posed integral equation of the first kind, and an efficient filtering technique is proposed for filtering the oscillation out. Although it is interesting to incorporate the filtering technique into the present method, it should be somewhat challenging since the flow solver is different, i.e., the present method is based on the LBM whereas their technique is for a Navier-Stokes solver.

575 Investigation on the cause of the spurious oscillation and a remedy for it remains in future work. 


\section{Conclusions}

We have proposed an immersed boundary-lattice Boltzmann method using the discontinuity of the stress tensor. In the present method, (i) the desired particle distribution functions which satisfy the no-slip condition on the boundary are calculated by using the bounce-back scheme, (ii) the stress tensors inside and outside the boundary are calculated by using the relation between the stress tensor and the particle distribution functions, and then (iii) the body force is determined by the relation between the body force and the discontinuity of the stress tensor. The present method has two advantages, i.e., the stress tensor on the boundary in the external fluid can be easily calculated, and the total force and torque acting on the boundary from the external fluid can be obtained without calculating the internal mass effect.

In order to validate the present method, we applied it to simulations of typical moving-boundary flows, i.e., a Taylor-Couette flow, an oscillating circular cylinder in a stationary fluid, the sedimentation of an elliptical cylinder, and the sedimentation of a sphere. As a result, it was found that the present method has the first-order spatial accuracy and has a good agreement with other numerical and experimental results.

In addition, we discussed two problems of the present method, i.e., penetration and spurious oscillation of local force, and a possible remedy for them. As for the penetration across the boundary, it was found that the preprocessing by the smoothed-profile method is effective for preventing the penetration. As for the spurious oscillation of the local force, we suggested that the cause of the spurious oscillation might be attributed to the bounce-back scheme in the lattice Boltzmann method or to the way to determine the body force in the immersed boundary method. Investigation on its cause and a remedy for it remains in future work.

\section{Acknowldegement}
This work was partially supported by JSPS KAKENHI Grant Number JP16K18012. 


\section{Appendix A. Nondimensional variables}

In Section 3, we use the following nondimensional variables defined by a characteristic length $\hat{H}_{0}$, a characteristic particle speed $\hat{c}$, a characteristic time scale $\hat{t}_{0}=\hat{H}_{0} / \hat{U}_{0}$ where $\hat{U}_{0}$ is a characteristic flow speed, and a fluid density $\hat{\rho}_{\mathrm{f}}$ :

$$
\left.\begin{array}{lll}
\boldsymbol{c}_{i}=\hat{\boldsymbol{c}}_{i} / \hat{c}, & \boldsymbol{x}=\hat{\boldsymbol{x}} / \hat{H}_{0}, & t=\hat{t} / \hat{t}_{0}, \\
\Delta x=\Delta \hat{x} / \hat{H}_{0}, & \Delta t=\Delta \hat{t} / \hat{t}_{0}, & c_{\mathrm{s}}=\hat{c}_{\mathrm{s}} / c, \\
f_{i}=\hat{f}_{i} / \hat{\rho}_{\mathrm{f}}, & \boldsymbol{u}=\hat{\boldsymbol{u}} / \hat{c}, & p=\hat{p} /\left(\hat{\rho}_{\mathrm{f}} \hat{c}^{2}\right), \\
\sigma_{\alpha \beta}=\hat{\sigma}_{\alpha \beta} /\left(\hat{\rho}_{\mathrm{f}} \hat{c}^{2}\right) & \nu=\hat{\nu} /\left(\hat{c} \hat{H}_{0}\right), & \boldsymbol{g}=\hat{\boldsymbol{g}} \hat{H}_{0} /\left(\hat{\rho}_{\mathrm{f}} \hat{c}^{2}\right), \\
\boldsymbol{X}_{k}=\hat{\boldsymbol{X}}_{k} / \hat{H}_{0}, & \boldsymbol{U}_{k}=\hat{\boldsymbol{U}}_{k} / \hat{U}_{0}, & \boldsymbol{P}=\hat{\boldsymbol{P}} /\left(\hat{\rho}_{\mathrm{f}} \hat{c}^{2}\right), \\
\boldsymbol{F}=\hat{\boldsymbol{F}} /\left(\hat{\rho}_{\mathrm{f}} \hat{c}^{2} \hat{H}_{0}^{2}\right), & \boldsymbol{T}=\hat{\boldsymbol{T}} /\left(\hat{\rho}_{\mathrm{f}} \hat{c}^{2} \hat{H}_{0}^{3}\right), & \\
\boldsymbol{X}_{\mathrm{c}}=\hat{\boldsymbol{X}}_{\mathrm{c}} / \hat{H}_{0}, & \boldsymbol{U}_{\mathrm{c}}=\hat{\boldsymbol{U}}_{\mathrm{c}} / \hat{c}^{\mathrm{B}}, & \boldsymbol{\Omega}_{\mathrm{c}}={ }^{\mathrm{B}} \hat{\boldsymbol{\Omega}}_{\mathrm{c}} \hat{H}_{0} / \hat{c}, \\
M=\hat{M} /\left(\hat{\rho}_{\mathrm{f}} \hat{H}_{0}^{3}\right), & \boldsymbol{I}_{\mathrm{B}}=\hat{\boldsymbol{I}}_{\mathrm{B}} /\left(\hat{\rho}_{\mathrm{f}} \hat{H}_{0}^{5}\right), & \alpha_{\mathrm{g}}=\hat{\alpha}_{\mathrm{g}} \hat{H}_{0} / \hat{c}^{2},
\end{array}\right\}
$$

where the circumflex represents 'dimensional.' It should be noted that the time step $\Delta \hat{t}$ is equal to the time span during which the particles travel one lattice spacing, that is, $\Delta \hat{x} / \Delta \hat{t}=\hat{c}$. We can easily obtain $\Delta t=S h \Delta x$ (where $\left.S h=\hat{H}_{0} /\left(\hat{t}_{0} \hat{c}\right)=\hat{U}_{0} / \hat{c}\right)$ from the above relation.

\section{Appendix B. Derivation of the stress tensor}

In this section, we derive the stress tensor given by Eq. (7). In order to derive the formulation, we have to perform an asymptotic analysis of the lattice Boltzmann equation. The following analysis is based on Refs. [25, 28, 64]. We starts the analysis from the formulation shown in Section 3.1. We set $c_{\mathrm{s}}=1 / \sqrt{3}$ in the equations used in the present analysis for simplicity. 
Appendix B.1. Moments of $E_{i}$

At first, we prepare the moments of $E_{i}$ as follows:

$$
\begin{aligned}
& \sum_{i=1}^{b} E_{i}=1 \\
& \sum_{i=1}^{b} E_{i} c_{i \alpha}=0 \\
& \sum_{i=1}^{b} E_{i} c_{i \alpha} c_{i \beta}=\frac{1}{3} \delta_{\alpha \beta} \\
& \sum_{i=1}^{b} E_{i} c_{i \alpha} c_{i \beta} c_{i \gamma}=0 \\
& \sum_{i=1}^{b} E_{i} c_{i \alpha} c_{i \beta} c_{i \gamma} c_{i \delta}=\frac{1}{9}\left(\delta_{\alpha \beta} \delta_{\gamma \delta}+\delta_{\alpha \gamma} \delta_{\beta \delta}+\delta_{\alpha \delta} \delta_{\beta \gamma}\right), \\
& \sum_{i=1}^{b} E_{i} c_{i \alpha} c_{i \beta} c_{i \gamma} c_{i \delta} c_{i \epsilon}=0
\end{aligned}
$$

where $\alpha, \beta, \gamma, \delta, \epsilon=x, y$ and $b=9$ for the D2Q9 model, and $\alpha, \beta, \gamma, \delta, \epsilon=x, y, z$ and $b=15$ for the D3Q15 model. It should be noted that the above moments has the same form independently of the dimensionality. The following analysis is independent of the dimensionality, too.

\section{Appendix B.2. Asymptotic analysis of the lattice Boltzmann equation}

We rewrite the lattice Boltzmann equation (3) by shifting the point $\boldsymbol{x}$ to $\boldsymbol{x}-\boldsymbol{c}_{i} \Delta x$ as follows:

$$
f_{i}(\boldsymbol{x}, t+\Delta t)=f_{i}\left(\boldsymbol{x}-\boldsymbol{c}_{i} \Delta x, t\right)-\frac{1}{\tau} g_{i}\left(\boldsymbol{x}-\boldsymbol{c}_{i} \Delta x, t\right),
$$

where

$$
g_{i}(\boldsymbol{x}, t)=f_{i}(\boldsymbol{x}, t)-f_{i}^{\mathrm{eq}}(p(\boldsymbol{x}, t), \boldsymbol{u}(\boldsymbol{x}, t)) .
$$

We write $\Delta x=\varepsilon$ for simplicity. We assume that the Mach number is $O(\varepsilon)$ and $\varepsilon \ll 1$. Since $\Delta t=S h \Delta x$ and $S h=O(\Delta x)$, we can write $\Delta t=B \varepsilon^{2}$ where $B=$ const. $=O(1)$. We express all terms in Eq. (B.7) as their Taylor expansions 
around $(\boldsymbol{x}, t)$ as follows:

$$
\begin{aligned}
f_{i}(\boldsymbol{x}, t+\Delta t) & =\sum_{n=0}^{\infty} \frac{1}{n !}\left(B \varepsilon^{2}\right)^{n} \frac{\partial^{n} f_{i}}{\partial t^{n}}(\boldsymbol{x}, t), \\
f_{i}\left(\boldsymbol{x}-\boldsymbol{c}_{i} \Delta x, t\right) & =\sum_{n=0}^{\infty} \frac{1}{n !}(-\varepsilon)^{n}\left(\boldsymbol{c}_{i} \cdot \boldsymbol{\nabla}\right)^{n} f_{i}(\boldsymbol{x}, t), \\
g_{i}\left(\boldsymbol{x}-\boldsymbol{c}_{i} \Delta x, t\right) & =\sum_{n=0}^{\infty} \frac{1}{n !}(-\varepsilon)^{n}\left(\boldsymbol{c}_{i} \cdot \boldsymbol{\nabla}\right)^{n} g_{i}(\boldsymbol{x}, t) .
\end{aligned}
$$

The solution of the lattice Boltzmann equation for small $\varepsilon$ is investigated in the form of the following asymptotic expansion:

$$
f_{i}=f_{i}^{(0)}+\varepsilon f_{i}^{(1)}+\varepsilon^{2} f_{i}^{(2)}+\varepsilon^{3} f_{i}^{(3)}+\cdots .
$$

We expand $f_{i}^{\text {eq }}$ and $g_{i}$ in the same way by

$$
\begin{aligned}
& f_{i}^{\mathrm{eq}}=f_{i}^{\mathrm{eq}(0)}+\varepsilon f_{i}^{\mathrm{eq}(1)}+\varepsilon^{2} f_{i}^{\mathrm{eq}(2)}+\varepsilon^{3} f_{i}^{\mathrm{eq}(3)}+\cdots, \\
& g_{i}=g_{i}^{(0)}+\varepsilon g_{i}^{(1)}+\varepsilon^{2} g_{i}^{(2)}+\varepsilon^{3} g_{i}^{(3)}+\cdots,
\end{aligned}
$$

where $g_{i}^{(k)}=f_{i}^{(k)}-f_{i}^{\text {eq }(k)}(k=0,1,2,3, \ldots)$. The macroscopic variables $p$ and $u_{\alpha}$ are expanded as follows:

$$
\begin{aligned}
& p=\frac{1}{3}+\varepsilon p^{(1)}+\varepsilon^{2} p^{(2)}+\varepsilon^{3} p^{(3)}+\cdots, \\
& u_{\alpha}=\varepsilon u_{\alpha}^{(1)}+\varepsilon^{2} u_{\alpha}^{(2)}+\varepsilon^{3} u_{\alpha}^{(3)}+\cdots .
\end{aligned}
$$

Since the Mach number is assumed to be $O(\varepsilon)$, i.e., the deviation from the equilibrium state at rest is $O(\varepsilon)$, the term of the order of $O(1)$ in $p$ is constant and the perturbation in $u_{\alpha}$ starts from the order of $O(\varepsilon)$. All the coefficients in the above expansion and their derivatives are assumed to be $O(1)$. By substituting Eqs. (B.15) and (B.16) into Eq. (4), we can write the coefficients in the expansion of $f_{i}^{\text {eq }}$ by those of $p$ and $u_{\alpha}$ as follows:

$$
\begin{aligned}
f_{i}^{\mathrm{eq}(0)} & =E_{i} \\
f_{i}^{\mathrm{eq}(1)} & =E_{i}\left[3 p^{(1)}+3 c_{i \alpha} u_{\alpha}^{(1)}\right] \\
f_{i}^{\mathrm{eq}(2)} & =E_{i}\left[3 p^{(2)}+3 c_{i \alpha} u_{\alpha}^{(2)}+\frac{9}{2} c_{i \alpha} c_{i \beta} u_{\alpha}^{(1)} u_{\beta}^{(1)}-\frac{3}{2} u_{\alpha}^{(1)} u_{\alpha}^{(1)}\right],
\end{aligned}
$$


where the summation convention is used. It should be noted that if a different equilibrium PDF is used alternatively, Eqs. (B.17)-(B.19) will be changed.

By substituting Eqs.(B.9)-(B.12) and (B.14) into Eq. (B.7) and by equating the terms of the same order of power of $\varepsilon$, we derive the following equations:

$$
\begin{aligned}
g_{i}^{(0)} & =0 \\
g_{i}^{(1)} & =c_{i \alpha} \frac{\partial g_{i}^{(0)}}{\partial x_{\alpha}}-\tau c_{i \alpha} \frac{\partial f_{i}^{(0)}}{\partial x_{\alpha}} \\
g_{i}^{(2)} & =c_{i \alpha} \frac{\partial g_{i}^{(1)}}{\partial x_{\alpha}}-\frac{1}{2} c_{i \alpha} c_{i \beta} \frac{\partial^{2} g_{i}^{(0)}}{\partial x_{\alpha} \partial x_{\beta}} \\
& -\tau\left[B \frac{\partial f_{i}^{(0)}}{\partial t}+c_{i \alpha} \frac{\partial f_{i}^{(1)}}{\partial x_{\alpha}}-\frac{1}{2} c_{i \alpha} c_{i \beta} \frac{\partial^{2} f_{i}^{(0)}}{\partial x_{\alpha} \partial x_{\beta}}\right] \\
g_{i}^{(3)} & =c_{i \alpha} \frac{\partial g_{i}^{(2)}}{\partial x_{\alpha}}-\frac{1}{2} c_{i \alpha} c_{i \beta} \frac{\partial^{2} g_{i}^{(1)}}{\partial x_{\alpha} \partial x_{\beta}}+\frac{1}{6} c_{i \alpha} c_{i \beta} c_{i \gamma} \frac{\partial^{3} g_{i}^{(0)}}{\partial x_{\alpha} \partial x_{\beta} \partial x_{\gamma}} \\
& -\tau\left[B \frac{\partial f_{i}^{(1)}}{\partial t}+c_{i \alpha} \frac{\partial f_{i}^{(2)}}{\partial x_{\alpha}}-\frac{1}{2} c_{i \alpha} c_{i \beta} \frac{\partial^{2} f_{i}^{(1)}}{\partial x_{\alpha} \partial x_{\beta}}+\frac{1}{6} c_{i \alpha} c_{i \beta} c_{i \gamma} \frac{\partial^{3} f_{i}^{(0)}}{\partial x_{\alpha} \partial x_{\beta} \partial x_{\gamma}}\right]
\end{aligned}
$$

By using the above equations and considering that $f_{i}^{\text {eq(0) }}$ is constant as shown in Eq. (B.17), we can write $f_{i}^{(k)}$ by the coefficients in the expansion of $f_{i}^{\text {eq }}$ as follows:

$$
\begin{aligned}
f_{i}^{(0)} & =f_{i}^{\mathrm{eq}(0)}, \\
f_{i}^{(1)} & =f_{i}^{\mathrm{eq}(1)}, \\
f_{i}^{(2)} & =f_{i}^{\mathrm{eq}(2)}-\tau c_{i \alpha} \frac{\partial f_{i}^{\mathrm{eq}(1)}}{\partial x_{\alpha}}, \\
f_{i}^{(3)} & =f_{i}^{\mathrm{eq}(3)}-\tau\left[B \frac{\partial f_{i}^{\mathrm{eq}(1)}}{\partial t}+c_{i \alpha} \frac{\partial f_{i}^{\mathrm{eq}(2)}}{\partial x_{\alpha}}+\left(\frac{1}{2}-\tau\right) c_{i \alpha} c_{i \beta} \frac{\partial^{2} f_{i}^{\mathrm{eq}(1)}}{\partial x_{\alpha} \partial x_{\beta}}\right] .
\end{aligned}
$$

From the orthogonality conditions $\sum_{i=i}^{b}\left(f_{i}-f_{i}^{\mathrm{eq}}\right)=\sum_{i=i}^{b} c_{i \alpha}\left(f_{i}-f_{i}^{\mathrm{eq}}\right)=0$, i.e., $\sum_{i=i}^{b}\left(f_{i}^{(k)}-f_{i}^{\mathrm{eq}(k)}\right)=\sum_{i=i}^{b} c_{i \alpha}\left(f_{i}^{(k)}-f_{i}^{\mathrm{eq}(k)}\right)=0(k=0,1,2,3, \ldots)$ [64], 
the partial differential equation (B.26) must satisfy the following conditions:

$$
\begin{aligned}
& \sum_{i=1}^{b}\left(-\tau c_{i \alpha} \frac{\partial f_{i}^{\mathrm{eq}(1)}}{\partial x_{\alpha}}\right)=0 \\
& \sum_{i=1}^{b} c_{i \alpha}\left(-\tau c_{i \beta} \frac{\partial f_{i}^{\mathrm{eq}(1)}}{\partial x_{\beta}}\right)=0 .
\end{aligned}
$$

Then, we have

$$
\begin{aligned}
& \frac{\partial}{\partial x_{\alpha}} \sum_{i=1}^{b} c_{i \alpha} f_{i}^{\mathrm{eq}(1)}=0 \\
& \frac{\partial}{\partial x_{\beta}} \sum_{i=1}^{b} c_{i \alpha} c_{i \beta} f_{i}^{\mathrm{eq}(1)}=0 .
\end{aligned}
$$

By substituting Eq. (B.18) into the above equations and by using Eqs. (B.1)(B.6), we derive the following equations:

$$
\begin{aligned}
& \frac{\partial u_{\alpha}^{(1)}}{\partial x_{\alpha}}=0, \\
& \frac{\partial p^{(1)}}{\partial x_{\alpha}}=0 .
\end{aligned}
$$

By applying the same analysis to Eq. (B.27) and by using Eqs. (B.32) and (B.33), we derive the following equations:

$$
\begin{aligned}
& B \frac{\partial p^{(1)}}{\partial t}+\frac{1}{3} \frac{\partial u_{\alpha}^{(2)}}{\partial x_{\alpha}}=0 \\
& B \frac{\partial u_{\alpha}^{(1)}}{\partial t}+\frac{\partial}{\partial x_{\beta}}\left(u_{\alpha}^{(1)} u_{\beta}^{(1)}\right)=-\frac{\partial p^{(2)}}{\partial x_{\alpha}}+\frac{1}{3}\left(\tau-\frac{1}{2}\right) \frac{\partial^{2} u_{\alpha}^{(1)}}{\partial x_{\beta}^{2}} .
\end{aligned}
$$

Appendix B.3. Stress tensor

By using the results of the asymptotic analysis shown in the previous section, we derive the stress tensor in terms of the PDF.

The definition of the stress tensor is given by

$$
\sigma_{\alpha \beta}=-p \delta_{\alpha \beta}+\mu\left(\frac{\partial u_{\alpha}}{\partial x_{\beta}}+\frac{\partial u_{\beta}}{\partial x_{\alpha}}\right),
$$

where $\mu$ is the viscosity given by

$$
\mu=\frac{1}{3} \rho_{\mathrm{f}}\left(\tau-\frac{1}{2}\right) \varepsilon,
$$


where $\rho_{\mathrm{f}}=1$ in the nondimensional form defined in Appendix A. In the following, we derive the second term of the right-hand side of Eq. (B.36) from the second moment of the PDF, i.e., $\sum_{i=1}^{b} c_{i \alpha} c_{i \beta} f_{i}$.

By substituting Eqs. (B.24)-(B.27) into Eq. (B.12), we have

$$
\begin{aligned}
f_{i}= & f_{i}^{\mathrm{eq}(0)}+\varepsilon f_{i}^{\mathrm{eq}(1)}+\varepsilon^{2}\left(f_{i}^{\mathrm{eq}(2)}-\tau c_{i \alpha} \frac{\partial f_{i}^{\mathrm{eq}(1)}}{\partial x_{\alpha}}\right) \\
& +\varepsilon^{3}\left[f_{i}^{\mathrm{eq}(3)}-\tau\left\{B \frac{\partial f_{i}^{\mathrm{eq}(1)}}{\partial t}+c_{i \alpha} \frac{\partial f_{i}^{\mathrm{eq}(2)}}{\partial x_{\alpha}}+\left(\frac{1}{2}-\tau\right) c_{i \alpha} c_{i \beta} \frac{\partial^{2} f_{i}^{\mathrm{eq}(1)}}{\partial x_{\alpha} \partial x_{\beta}}\right\}\right]+O\left(\varepsilon^{4}\right) .
\end{aligned}
$$

Considering Eq. (B.13), we have

$$
\begin{aligned}
f_{i}= & f_{i}^{\mathrm{eq}}-\varepsilon^{2} \tau c_{i \alpha} \frac{\partial f_{i}^{\mathrm{eq}(1)}}{\partial x_{\alpha}} \\
& -\varepsilon^{3} \tau\left[B \frac{\partial f_{i}^{\mathrm{eq}(1)}}{\partial t}+c_{i \alpha} \frac{\partial f_{i}^{\mathrm{eq}(2)}}{\partial x_{\alpha}}+\left(\frac{1}{2}-\tau\right) c_{i \alpha} c_{i \beta} \frac{\partial^{2} f_{i}^{\mathrm{eq}(1)}}{\partial x_{\alpha} \partial x_{\beta}}\right]+O\left(\varepsilon^{4}\right) .
\end{aligned}
$$

Therefore, the second moment of the PDF reduces to

$$
\begin{aligned}
\sum_{i=1}^{b} c_{i \alpha} c_{i \beta} f_{i}= & \sum_{i=1}^{b} c_{i \alpha} c_{i \beta} f_{i}^{\mathrm{eq}}-\varepsilon^{2} \tau \frac{\partial}{\partial x_{\gamma}} \sum_{i=1}^{b} c_{i \alpha} c_{i \beta} c_{i \gamma} f_{i}^{\mathrm{eq}(1)} \\
& -\varepsilon^{3} \tau\left[B \frac{\partial}{\partial t} \sum_{i=1}^{b} c_{i \alpha} c_{i \beta} f_{i}^{\mathrm{eq}(1)}+\frac{\partial}{\partial x_{\gamma}} \sum_{i=1}^{b} c_{i \alpha} c_{i \beta} c_{i \gamma} f_{i}^{\mathrm{eq}(2)}\right. \\
& \left.+\left(\frac{1}{2}-\tau\right) \frac{\partial^{2}}{\partial x_{\gamma} \partial x_{\delta}} \sum_{i=1}^{b} c_{i \alpha} c_{i \beta} c_{i \gamma} c_{i \delta} f_{i}^{\mathrm{eq}(1)}\right]+O\left(\varepsilon^{4}\right) .
\end{aligned}
$$

By substituting Eqs. (4), (B.18), and (B.19) into the above equation and by using Eqs. (B.1)-(B.6), we derive the following equations:

$$
\begin{aligned}
\sum_{i=1}^{b} c_{i \alpha} c_{i \beta} f_{i}= & p \delta_{\alpha \beta}+u_{\alpha} u_{\beta}-\varepsilon^{2} \tau \frac{1}{3}\left(\frac{\partial u_{\alpha}^{(1)}}{\partial x_{\beta}}+\frac{\partial u_{\beta}^{(1)}}{\partial x_{\alpha}}+\frac{\partial u_{\gamma}^{(1)}}{\partial u_{\gamma}} \delta_{\alpha \beta}\right) \\
& -\varepsilon^{3} \tau\left[\left(B \frac{\partial p^{(1)}}{\partial t}+\frac{1}{3} \frac{\partial u_{\gamma}^{(2)}}{\partial x_{\gamma}}\right) \delta_{\alpha \beta}\right. \\
& \left.+\frac{1}{3}\left(\frac{\partial u_{\alpha}^{(2)}}{\partial x_{\beta}}+\frac{\partial u_{\beta}^{(2)}}{\partial x_{\alpha}}\right)+\left(\frac{1}{2}-\tau\right) \frac{\partial^{2} p^{(1)}}{\partial x_{\alpha} \partial x_{\beta}}\right]+O\left(\varepsilon^{4}\right) .
\end{aligned}
$$


By using Eqs. (B.32)-(B.34), we have

$$
\begin{aligned}
\sum_{i=1}^{b} c_{i \alpha} c_{i \beta} f_{i}= & p \delta_{\alpha \beta}+u_{\alpha} u_{\beta}-\varepsilon^{2} \tau \frac{1}{3}\left(\frac{\partial u_{\alpha}^{(1)}}{\partial x_{\beta}}+\frac{\partial u_{\beta}^{(1)}}{\partial x_{\alpha}}\right) \\
& -\varepsilon^{3} \tau \frac{1}{3}\left(\frac{\partial u_{\alpha}^{(2)}}{\partial x_{\beta}}+\frac{\partial u_{\beta}^{(2)}}{\partial x_{\alpha}}\right)+O\left(\varepsilon^{4}\right) .
\end{aligned}
$$

Considering Eq. (B.16), we have

$$
\sum_{i=1}^{b} c_{i \alpha} c_{i \beta} f_{i}=p \delta_{\alpha \beta}+u_{\alpha} u_{\beta}-\frac{1}{3} \tau \varepsilon\left(\frac{\partial u_{\alpha}}{\partial x_{\beta}}+\frac{\partial u_{\beta}}{\partial x_{\alpha}}\right)+O\left(\varepsilon^{4}\right) .
$$

Therefore, the following relation can be obtained:

$\sum_{i=1}^{b}\left(c_{i \alpha}-u_{\alpha}\right)\left(c_{i \beta}-u_{\beta}\right) f_{i}=p \delta_{\alpha \beta}+(3 p-1) u_{\alpha} u_{\beta}-\frac{1}{3} \tau \varepsilon\left(\frac{\partial u_{\alpha}}{\partial x_{\beta}}+\frac{\partial u_{\beta}}{\partial x_{\alpha}}\right)+O\left(\varepsilon^{4}\right)$.

Finally, from Eqs. (B.36), (B.37), and (B.44), we can obtain

$\sigma_{\alpha \beta}=-\frac{1}{2 \tau} p \delta_{\alpha \beta}-\frac{\tau-1 / 2}{\tau}\left[\sum_{i=1}^{b} f_{i}\left(c_{i \alpha}-u_{\alpha}\right)\left(c_{i \beta}-u_{\beta}\right)-(3 p-1) u_{\alpha} u_{\beta}\right]+O\left(\varepsilon^{4}\right)$.

\section{References}

\section{References}

[1] A. J. C. Ladd, Numerical simulations of particulate suspensions via a discretized Boltzmann equation. Part 1. Theoretical foundation, J. Fluid Mech. 271 (1994) 285-309.

[2] A. J. C. Ladd, Numerical simulations of particulate suspensions via a discretized Boltzmann equation. Part 2. Numerical results, J. Fluid Mech. 271 (1994) 311-339.

[3] C. S. Peskin, Flow patterns around heart valves: A numerical method, J. 
[4] C. S. Peskin, Numerical analysis of blood flow in the heart, J. Comput. Phys. 25 (1977) 220-252.

[5] R. Mittal, G. Iaccarino, Immersed boundary methods, Annu. Rev. Fluid Mech. 37 (2005) 239-261.

[6] M.-C. Lai, C. S. Peskin, An immersed boundary method with formal second-order accuracy and reduced numerical viscosity, J. Comput. Phys. 160 (2000) 705-719.

[7] E. Arquis, J.-P. Caltagirone, Sur les conditions hydrodynamiques au voisinage d'une interface milieu fluide - milieux poreux: application à la convection naturelle, Comptes Rendus de l'Academie des Sciences Paris II 299 (1984) $1-4$.

[8] D. Goldstein, R. Handler, L. Sirovich, Modeling a no-slip flow boundary with an external force field, J. Comput. Phys. 105 (1993) 354-366.

[9] J. Mohd-Yusof, Combined immersed-boundary/B-spline methods for simulations of flow in complex geometries, in: CTR Annual Research Briefs, NASA Ames/Stanford University, 1997, pp. 317-327.

[10] R. Glowinski, T.-W. Pan, T. Hesla, D. Joseph, A distributed Lagrange multiplier/fictitious domain method for particulate flows, Int. J. Multiphase Flow 25 (1999) 755-794.

[11] H. S. Udaykumar, W. Shyy, M. M. Rao, Elafint: A mixed EulerianLagrangian method for fluid flows with complex and moving boundaries, Int. J. Numer. Methods Fluids 22 (1996) 691-705.

[12] S. Majumdar, G. Iaccarino, P. Durbin, RANS solvers with adaptive structured boundary non-conforming grids, in: Annu. Res. Briefs 2001, Cent. Turbul. Res., 2001, pp. 353-366.

[13] C. S. Peskin, The immersed boundary method, Acta Numerica 11 (2002) 479-517. 
[14] M. Uhlmann, An immersed boundary method with direct forcing for the simulation of particulate flows, J. Comput. Phys. 209 (2005) 448-476.

[23] Y. Hu, D. C. Li, S. Shu, X. D. Niu, Modified momentum exchange method for fluid-particle interactions in the lattice Boltzmann method, Phys. Rev. E 91 (2015) 033301 (14pp). 
[24] Y. Hu, D. C. Li, S. Shu, X. D. Niu, Study of multiple steady solutions for the 2D natural convection in a concentric horizontal annulus with a constant heat flux wall using immersed boundary-lattice Boltzmann method, Int. J. Heat Mass Transfer 81 (2015) 591-601.

[25] T. Inamuro, K. Maeba, F. Ogino, Flow between parallel walls containing the lines of neutrally buoyant circular cylinders, Int. J. Multiphase Flow 26 (2000) 1981-2004.

[26] K. Suzuki, T. Inamuro, Effect of internal mass in the simulation of a moving body by the immersed boundary method, Comput. Fluids 49 (2011) 173187.

[27] X. He, L.-S. Luo, Lattice Boltzmann model for the incompressible NavierStokes equation, J. Stat. Phys. 88 (1997) 927-944.

[28] T. Inamuro, M. Yoshino, F. Ogino, Accuracy of the lattice Boltzmann method for small Knudsen number with finite Reynolds number, Phys. Fluid 9 (1997) 3535-3542.

[29] M. Junk, A. Klar, L.-S. Luo, Asymptotic analysis of the lattice Boltzmann equation, J. Comput. Phys. 210 (2005) 676-704.

[30] Z. Guo, C. Zhang, B. Shi, Discrete lattice effects on the forcing term in the lattice Boltzmann method, Phys. Rev. E 65 (2002) 046308 (6pp).

[31] X. He, Q. Zou, L. S. Luo, M. Dembo, Analytic solutions of simple flows and analysis of noslip boundary conditions for the lattice Boltzmann BGK model, Phys. Rev. E 87 (1997) 115-136.

[32] Z. Wang, J. Fan, K. Luo, Combined multi-direct forcing and immersed boundary method for simulating flows with moving particles, Int. J. Multiphase Flow 34 (2008) 283-302.

[33] K. Suzuki, I. Okada, M. Yoshino, Accuracy of the laminar boundary layer on a flat plate in an immersed boundary-lattice Boltzmann simulation, J. Fluid Sci. Technol. 11 (2016) 16-00488 (17pp). 
[34] K. Suzuki, M. Yoshino, Numerical simulations for aerodynamic performance of a butterfly-like flapping wing-body model with various wing planforms, Commun. Comput. Phys. 23 (2018) 951-979.

${ }_{725}$ [35] D. Yu, R. Mei, L. S. Luo, W. Shyy, Viscous flow computations with the method of lattice Boltzmann equation, Prog. Aerosp. Sci. 39 (2003) 329367.

[36] P. Lallemand, L. S. Luo, Lattice Boltzmann method for moving boundaries, J. Comput. Phys. 184 (2003) 406-421.

[37] A. ten Cate, C. H. Nieuwstad, J. J. Derksen, H. E. A. Van den Akker, Particle imaging velocimetry experiments and lattice-Boltzmann simulations on a single sphere settling under gravity, Phys. Fluids 14 (2002) 4012-4025.

[38] Z. G. Feng, E. E. Michaelides, Robust treatment of no-slip boundary condition and velocity updating for the lattice-Boltzmann simulation of particulate flows, Comput. Fluids 38 (2009) 370-381.

[39] L. Shen, E. Chan, P. Lin, Calculation of hydrodynamic forces acting on a submerged moving object using immersed boundary method, Comput. Fluids 38 (2009) 691-702.

[40] R. D. Guy, A. A. Hartenstine, On the accuracy of direct forcing immersed boundary methods with projection methods, J. Comput. Phys. 229 (2010) 2479-2496.

[41] K. Suzuki, T. Inamuro, A higher-order immersed boundary-lattice Boltzmann method using a smooth velocity field near boundaries, Comput. Fluids 76 (2013) 105-115.

[42] N.-Q. Nguyen, A. J. C. Ladd, Lubrication corrections for lattice-Boltzmann simulations of particle suspensions, Phys. Rev. E 66 (2002) 046708 (12pp).

[43] H. Dütsch, F. Durst, S. Becker, H. Lienhart, Low-Reynolds-number flow around an oscillating circular cylinder at low Keulegan-Carpenter numbers, J. Fluid Mech. 360 (1998) 249-271. 
[52] X. He, G. Doolen, Lattice Boltzmann method on curvilinear coordinates system: flow around a circular cylinder, J. Comput. Phys. 134 (2009) 306315.

[53] K. Taira, T. Colonius, The immersed boundary method: A projection ap775

[44] Z. Xia, K. W. Connington, S. Rapaka, P. Yue, J. J. Feng, S. Chen, Flow patterns in the sedimentation of an elliptical particle, J. Fluid Mech. 625 (2009) 249-272.

[45] F. X. Giraldo, Lagrange-Galerkin methods on spherical geodesic grid, J. Comput. Phys. 136 (1997) 197-213.

[46] Z. G. Feng, E. E. Michaelides, Proteus: a direct forcing method in the simulations of particulate flows, J. Comput. Phys. 202 (2005) 20-51.

[47] C.-C. Liao, Y.-W. Cang, C.-A. Lin, J. M. McDonough, Simulating flows with moving rigid boundary using immersed-boundary method, Comput. Fluids 39 (2010) 152-167.

[48] J. Wu, C. Shu, Implicit velocity correction-based immersed boundarylattice Boltzmann method and its applications, J. Comput. Phys. 228 (2009) 1963-1979.

[49] D. J. Tritton, Experiments on the flow past a circular cylinder at low Reynolds number, J. Fluid Mech. 6 (1959) 547-567.

50] M. Coutanceau, R. Bouard, Experimental determination of the main features of the viscous flow in the wake of a circular cylinder in uniform translation, J. Fluid Mech. 79 (1977) 231-256.

[51] S. C. R. Deniss, G. Cheng, Numerical solutions for steady flow past a circular cylinder at Reynolds number up to 100, J. Fluid Mech. 42 (1970) 471-489.

proach, J. Comput. Phys. 225 (2007) 2118-2137. 
[54] A. Roshko, Report 1191: On the development of turbulent wakes from vortex streets, NACA, 1954.

[55] C. Liu, X. Zheng, C. H. Sung, Preconditioned multigrid methods for unsteady incompressible flows, J. Comput. Phys. 139 (1998) 35-57.

[56] M. N. Linnick, H. F. Fasel, A high-order immersed interface method for simulating unsteady incompressible flows on irregular domains, J. Comput. Phys. 204 (2005) 157-192.

[57] Y. Wang, C. Shu, C. J. Teo, J. Wu, An immersed boundary-lattice Boltzmann flux solver and its applications to fluid-structure interaction problems, J. Fluid Struct. 54 (2015) 440-465.

[58] Y. Nakayama, R. Yamamoto, Simulation method to resolve hydrodynamic interactions in colloidal dispersions, Phys. Rev. E 71 (2005) 036707 (7 pp).

[59] S. Jafari, R. Yamamoto, M. Rahnama, Lattice-Boltzmann method combined with smoothed-profile method for particulate suspensions, Phys. Rev. E 83 (2011) 026702 (12 pp).

[60] Y. Mino, H. Shinto, S. Sakai, H. Matsuyama, Effect of internal mass in the lattice Boltzmann simulation of moving solid bodies by the smoothedprofile method, Phys. Rev. E 95 (2017) 043309 (10 pp).

[61] J. Park, K. Kwon, H. Choi, Numerical solutions of flow past a circular cylinder at Reynolds number up to 160, KSME Int. J. 12 (1998) 12001205.

[62] R. Mei, D. Yu, W. Shyy, Force evaluation in the lattice Boltzmann method involving curved geometry, Phys. Rev. E 65 (2002) 041203 (14pp).

[63] A. Goza, S. Liska, B. Morley, T. Colonius, Accurate computation of surface stresses and forces with immersed boundary methods, J. Comput. Phys. 321 (2016) 860-873. 
[64] P. Asinari, T. Ohwada, Connection between kinetic methods for fluiddynamic equations and macroscopic finite-difference schemes, Comput. Math. Appl. 58 (2009) 841-861. 